\title{
Adaptive Multiple-Model Switching Control of Robotic Manipulators
}

\author{
A Thesis \\ Presented to \\ the Faculty of the School of Engineering and Applied Science \\ UNIVERSITY OF VIRGINIA \\ In Partial Fulfillment \\ of the Requirements for the Degree \\ Master of Science in Electrical Engineering \\ by \\ JINGJING HAO \\ Dec. 2016
}




\section{APPROVAL SHEET}

This thesis is submitted in partial fulfillment of the

requirements for the degree of

Master of Science in Electrical Engineering

Jingjing Hao, Author

This thesis has been read and approved by the examining committee:

Professor Gang Tao, Thesis Advisor

Professor Zongli Lin

Professor Maite Brandt-Pearce

Accepted for the School of Engineering and Applied Science:

Dean, School of Engineering and Applied Science

Dec. 2016 


\section{Acknowledgments}

I would like to thank the many people who helped during the preparation of this thesis. I would first like to thank my thesis advisor Professor Gang Tao of the School of Engineering and Applied Science at University of Virginia. His wisdom and deep insights in control area steered me in the right direction during my research, and he was always willing to help whenever I could not independently figure out a problem.

I would also like to acknowledge two PhD students in Professor Tao's group, Ge Song and Thummaros Rugthum. I am gratefully indebted to them for their very valuable guidance and suggestions on this thesis.

Finally, I must express my very profound gratitude to my parents and to my friends for providing me with unfailing support and continuous encouragement throughout my years of study and through the process of researching and writing this thesis. This accomplishment would not have been possible without them. Thank you. 


\section{Abstract}

The control problem of robotic manipulators has drawn increasing attention during the past twenty years. Adaptive control, with its great potential for dealing with systems in uncertain environments, becomes a powerful tool in this area. Existing research focuses more on the convergence theorems, however, few literature concerns about the overall system performance.

This thesis first proposes a new dynamic prediction error based adaptive controller for robotic manipulators with uncertain parameters. Unlike most prediction errors used in the robotics literature, a dynamic prediction error is generated from an adaptive predictor of a parametrized and dynamic manipulator model. A multiplemodel adaptive control scheme is then developed using multiple prediction errors and multiple controllers, incorporated with multiple parameter estimators and a control switching mechanism. The use of an adaptive dynamic predictor for parameter estimation leads to a new, effective and simple control structure. Multiple controllers are constructed with different parameter estimators, and a most appropriate control signal is selected by the control switching mechanism which is designed to find the model that best approximates the manipulator dynamics. Closed-loop system stability and output tracking are proved and the detailed analysis is given. Simulation results demonstrate the desired control system performance. 


\section{Contents}

1 Introduction 1

1.1 Research Motivation . . . . . . . . . . . . . . . 2

1.2 Literature Review . . . . . . . . . . . . . . . . . . . 3

1.3 Thesis Outline . . . . . . . . . . . . . . . . 6

$\begin{array}{llr}2 & \text { Overview } & 8\end{array}$

2.1 Manipulator System Model and Control Objective . . . . . . . . . . . 8

2.2 Direct Adaptive Control Design . . . . . . . . . . . . . . . . 12

2.2.1 Design with Knowledge of the Acceleration $\ddot{q}(t)$. . . . . . . . 12

2.2.2 Design without Knowledge of the Acceleration $\ddot{q}(t) \ldots$. . . . . 14

2.3 Indirect Adaptive Control Design . . . . . . . . . . . . . . . . 16

2.3.1 Design with Knowledge of the Acceleration $\ddot{q}(t) \ldots$. . . . . . 16

2.3.2 Design without Knowledge of the Acceleration $\ddot{q}(t) \ldots 18$

2.4 Multiple-Model Control . . . . . . . . . . . . . . . . . . 22

2.4.1 General Methodology . . . . . . . . . . . . . . 22

2.4.2 Multiple-Model Design of Indirect Adaptive Control . . . . . . 26

2.5 Problem Statement . . . . . . . . . . . . . . . . . . . . 29

3 A Dynamic Prediction Error Based Adaptive Control Scheme 31

3.1 Nominal Controller . . . . . . . . . . . . . . . . . . . . 31 
3.2 Adaptive Parameter Estimation Design . . . . . . . . . . . . . . 33

3.2 .1 Parameter Estimation . . . . . . . . . . . . . . 33

3.2 .2 Compact Parametrization of $D(q) \dot{\hat{s}} \ldots \ldots \ldots \ldots$

3.2.3 A Parameter Projection Scheme . . . . . . . . . . . . . . 40

3.3 Adaptive Controller . . . . . . . . . . . . . . . . . . . . . . . . 42

3.4 Simulation Study . . . . . . . . . . . . . . . . . . . . . 44

3.4 .1 Simulation System _. . . . . . . . . . . . . . 44

3.4 .2 Simulation Results . . . . . . . . . . . . . . . 47

3.5 Simulation Discussion and Summary . . . . . . . . . . . 52

4 A Multiple-Model Adaptive Control Scheme 53

4.1 Problem Statement . . . . . . . . . . . . . . . . . . 53

4.2 Multiple-Model Adaptive Design . . . . . . . . . . . . . . . . 54

4.3 Adaptive Control System Analysis . . . . . . . . . . . . . . . 58

4.4 Comparison Study . . . . . . . . . . . . . . . . . . . . 61

4.5 Simulation Study . . . . . . . . . . . . . . . . 63

4.5.1 Simulation Results . . . . . . . . . . . . . . . . 64

4.5.2 Additional Simulation Results . . . . . . . . . . . . 70

5 Conclusions and Future Work $\quad 73$

5.1 Conclusions . . . . . . . . . . . . . . . . . . . 73

5.2 Future Research Topics . . . . . . . . . . . . . . . . . . . . . 74 


\section{List of Figures}

3.1 Tracking errors $e(t)=q(t)-q_{d}(t)$ for the single-model scheme (Case I). 49

3.2 Parameter errors of $\theta(t)$ for the single-model scheme (Case I). . . . . 49

3.3 Parameter errors of $\theta_{D}(t)$ and $\theta_{C}(t)$ for the single-model scheme (Case I). ................................ 50

3.4 Tracking errors $e(t)=q(t)-q_{d}(t)$ for the single-model scheme (Case II). 50

3.5 Parameter errors of $\theta(t)$ for the single-model scheme (Case II). . . . . 51

3.6 Parameter errors of $\theta_{D}(t)$ and $\theta_{C}(t)$ for the single-model scheme (Case II) . . . . . . . . . . . . . . . . . . . . . . . . . 51

4.1 Tracking errors $e(t)=q(t)-q_{d}(t)$ for the multiple-model scheme (Case

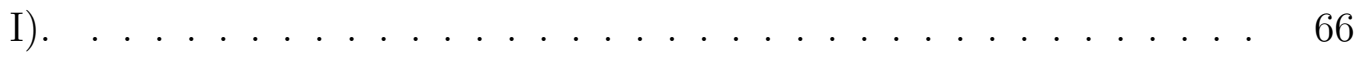

4.2 Parameter errors of $\theta_{(i)}(t)$ for the multiple-model scheme (Case I). . . 66

4.3 Parameter errors of $\theta_{D(i)}(t)$ and $\theta_{C(i)}(t)$ for the multiple-model scheme (Case I). . . . . . . . . . . . . . . . . . . . . . . . . . . . . . . 67

4.4 Control switching index $i$ and cost functions $J_{i}(t)$ of the multiple-model scheme $($ Case I). . . . . . . . . . . . . . . . . .

4.5 Tracking errors $e(t)=q(t)-q_{d}(t)$ for the multiple-model scheme (Case II) . . . . . . . . . . . . . . . . . . . . . . . 68

4.6 Parameter errors of $\theta_{(i)}(t)$ for the multiple-model scheme (Case II). . 68 
4.7 Parameter errors of $\theta_{D(i)}(t)$ and $\theta_{C(i)}(t)$ for the multiple-model scheme (Case II). . . . . . . . . . . . . . . . . . . . . . . . . . . 69

4.8 Control switching index $i$ and cost function $J_{i}(t)$ of the multiple-model scheme $($ Case II). . . . . . . . . . . . . . . . . . . . . . . . . . 69

4.9 Tracking error $e(t)=q(t)-q_{d}(t)$ for multiple-model scheme case III. . $\quad 71$

4.10 Control switching index $i$ and cost function $J_{i}(t)$ of multiple-model scheme case III. . . . . . . . . . . . . . . . . . . . . . . . . . . 72 


\section{Chapter 1}

\section{Introduction}

In the past two decades, unprecedented development in sensing, communications and computation has turned plenty of impossible ideas into real. Innovative technologies grow fast, changing the way we collecting and processing information. Robots, as a combination of knowledge from different fields such as mechanics, electronics, sensing and computing, have stimulated much interest among researchers. Important scientific results have emerged regarding to different aspects of robots.

The control problem has always been one of the most essential aspects in research related to robotic manipulators. Being an important methodology in control area, adaptive control is frequently considered to be a solution to this problem. In this thesis, we comprehensively discuss the adaptive control of robotic manipulators, and combine multiple-model control method with the existing control scheme to improve control effect. We will explore different aspects of the robotic manipulator's control problem in the following chapters. 


\subsection{Research Motivation}

Since the number of robotic manipulators used in industry increases significantly these years, control methods of manipulators have become important research areas. Different control methods have been considered in order to obtain better control effect under different circumstances. One of the most important characteristics of robot manipulators, which makes them difficult to control, is the high nonlinearity of their dynamics. Although it is possible to design a controller to achieve good performance if we known a precise model of the system [11], this controller is not capable of handling any changes and uncertainties in the system. However, a robot manipulator often has to interact with different environments such as grabbing or carrying different objects with unknown mass, dimensions, or orientations, which consequently makes it impractical to identify system parameters with sufficient accuracy at every circumstance.

When dealing with system uncertainties, adaptive control lends itself a powerful tool, and the problem of designing adaptive control laws for rigid robot manipulators that ensure asymptotic trajectory tracking has interested researchers for many years. The basic idea of adaptive control is to update the values of adaptive gains or parameters in the control laws according to some on-line algorithms corresponding to the changes and uncertainties happening to the system. The adaptive controller keeps updating itself until desired control objective is achieved. This control approach is especially useful for robot manipulators, since they usually execute repetitive tasks, and the tracking performance of adaptive controllers can be improved with time through the adaptation for each successive operation [37].

Since in all control algorithms, the convergence theorems are based on asymptotic arguments, very little is said about the transient behavior of the resulting system [7]. 
This is partly due to the fact that these algorithms are required to satisfy performance criteria, which are themselves asymptotic in nature. When large load variations happen, the transient tracking errors can be large and oscillatory, leading to poor overall performance. This is obviously an undesirable situation, especially when tracking requirements are stringent. Therefore, more research should be conducted to change this circumstance, and multiple-model control may be one of the solutions.

\section{$1.2 \quad$ Literature Review}

This section is concerned with current contributions to the research of robot control problem, including adaptive control of robotic manipulators, multiple-model and switching, and multiple-model control of robotic manipulators. Our goal is to provide a complete introduction to the most important concepts and research results in these subjects, and lay the foundation for the following work and discussion.

Adaptive control of robotic manipulators. In the literature related to adaptive robot control, many algorithms have been proposed these years. According to the signals researchers use to generate the parameter update laws, these algorithms can be classified into three categories: direct, indirect, and composite adaptive control.

The direct adaptive control algorithms drive parameter update laws using the tracking error, which is the error between desired output and actual output. Slotine and Li proposed a direct adaptive control algorithm in 1986, in which the global tracking convergence of an adaptive feedforward-plus-PD controller is established [33]. In the same year, Craig and others also proposed a direct adaptive controller based on computed-torque control and showed its global convergence [9], but this algorithm requires both measurements of the joint acceleration and modification of the adaptive algorithm to insure boundedness of the inverse of the estimated inertia matrix. In 
1987, Kelly and others proposed an adaptive impedance control algorithm, which removes the requirement on boundedness of the estimated inertia matrix [14]. Ortega and Spong proposed an adaptive motion controller with a new parameter update law in 1988, which is also capable of eliminating the requirement of boundedness of the estimated inertia matrix [31].

The indirect adaptive control algorithms, on the other hand, use the prediction error to generate update laws, which is the error between estimated parameter and true parameter. Middleton and Goodwin designed an indirect adaptive control algorithm composed of a modified computed-torque controller and a modified least-square estimator, and proved the global tracking convergence of this controller [22]. This algorithm does not require the measurement of the joint acceleration, but still need boundedness of the inverse of the estimated inertia matrix. Slotine and Li developed a globally convergent indirect adaptive controller in 1989, which removes the boundedness requirement [21].

The composite adaptive control algorithm uses both tracking error in the joint motion and prediction error in the predicted filtered torque to drive the parameter update laws. Proposed by Slotine and Li in 1987 [34], [35], the algorithm is based on the observation that the parameter uncertainty is reflected in both tracking error and prediction error, hence it is preferable to get the information from both sources. This algorithm also creates an automatic way of modulating the adaptive gains according to the excitation of the desired trajectory.

Multiple-model and switching. While many adaptive robot controllers have been proposed in the literature, tracking error convergence in these adaptive control algorithms are based on asymptotic arguments, as a result, the transient tracking errors may be quite large and oscillatory under large parametric uncertainties. For the purpose of improving overall tracking performance, adaptive control using multiple- 
model and switching is proposed. It is a general methodology for the design of adaptive control systems which can learn to operate efficiently in dynamical environments prossessing a high degree of uncertainty [24]. By describing the different environments using multiple models and switching to an appropriate controller followed by tuning or adaptation, the control effect would be efficiently improved. Multiple-model and switching is a rapidly growing area which has been investigated in detail since 1991. Researchers extended this methodology to both linear and nonlinear systems. In linear adaptive control, researchers aimed to develop stable and efficient switching schemes to obtain better transient response [25], [26]. In nonlinear systems, most research was focused on identifying and controlling dynamical systems using neural networks when only inputs and outputs are accessible [18], [19], [27].

The use of multiple-model and switching is not new in control theory. There are mainly three methods of adaptive control using multiple-model. The first is indirect adaptive control using multiple models, which was originally used by Middleton in 1988 [22]. These results were extended to adaptive control of discrete-time systems using multiple models by Narendra in 1998 [28] and the global stability in this case was proved in 2000 [29]. The second is direct adaptive control using multiple models. In 1986, Fu developed this type of controller for adaptive stabilization without a minimum-phase assumption and without knowledge of the sign of the high-frequency gain [12]. The third is weighted multipel models control, and this controller structure was came up with by Badr and Binder in discrete-time systems in 1985 [2]. This concept was extended to MIMO systems [1] and was later applied to a nonlinear thermal process [3].

Multiple-model control of robotic manipulators. Multiple-model and switching could attain its full potential in adaptive control of robot manipulators, because tracking performance of rigid robot manipulators is mainly determined by the accu- 
racy of initial values of parameters estimates and the choices of adaptive gains. When executing tasks with uncertainty, such as grabbing or carrying different objects with unknown masses, dimension, or gripping points, these parameters may vary greatly. If a single model scheme is used, the robot control system will have to adapt itself to a new environment every time system parameters change before appropriate control action can be taken. The slowness of the adaptation may result in a large transient error. Multiple-model and switching will allow us not only to identify the different environments, but also to control them rapidly [24].

In 1994, Ciliz firstly combined multiple-model and switching with robot manipulators, and proved the effect of this methodology in practical situations [5], [7]. Some experimental tests were successfully carried out on a two-link SCARA type direct drive arm in 1995 [6]. Ciliz utilized an adaptive control scheme which eliminated the acceleration vector by a first-order strictly proper filter (proposed by Middleton in 1988 [23]). To compensate for the effect brought by the filter, two items were added to structure of the controller, making it complicated and hard for calculation. Also, the positive definite and invertible property of the estimate of inertia matrix has not been proved. In this thesis, we will introduce a new simple prediction-based algorithm for adaptive robot control using multiple-model and switching to solve these problems.

\subsection{Thesis Outline}

This thesis proposes a new prediction-based adaptive control scheme for robotic manipulators, which does not need the measurement of acceleration information or compensation terms in the control input signal. The guarantee of positive definite and invertible property of the inertia matrix will also be implemented in this thesis. We will combine this scheme with multiple-model control and switching to solve the 
undesired transient problem, and conduct simulation to verify the enhancement of this new algorithm.

The content of this thesis is organized as follows.

In Chapter 2, we introduce some existing algorithms of adaptive robot control, which represent the development and main contributions at this stage. Some basic background information about multiple-model control is also presented here as an overview for this research area.

In Chapter 3, we develop the innovative adaptive robot control scheme using single-model control to deal with system uncertainties. Stability analysis is provided to demonstrate the effectiveness of our control scheme, and simulation results are discussed subsequently to analyze its performance.

For improvement of the system performance, this single-model control scheme is expanded to a multiple-model control scheme in Chapter 4. Necessarily, stability analysis of the adaptive multiple-model control system is also completed here. In order to help readers better understand our design, comparison study is provided, together with supportive simulation results under different cases.

Finally, conclusions for this thesis and potential future work are discussed in Chapter 5. The contributions this thesis have made are presented in this chapter. 


\section{Chapter 2}

\section{Overview}

Before starting to discuss the adaptive control of robotic manipulators based on multiple-model and switching, an overview of the previous results in adaptive robot control will be presented. In this chapter, a system model will be introduced first. Then, a discussion of four existing algorithms will follow, with two for direct adaptive control, and two for indirect adaptive control. The background knowledge of multiplemodel control and switching will also be introduced in the last section of this chapter.

\subsection{Manipulator System Model and Control Ob- jective}

To derive the dynamic equations of the robotic manipulator, we may refer to the Euler-Lagrange equations for a rigid-body mechanical system [37]:

$$
\frac{d}{d t} \frac{\partial L}{\partial \dot{q}}-\frac{\partial L}{\partial q}=u
$$


where $q=\left[q_{1}, \cdots, q_{n}\right]^{T}$ is the vector of position variables of $n$ joints of the robotic manipulator, $u=\left[u_{1}, \cdots, u_{n}\right]^{T}$ is the applied joint torque, and $L$ is the Lagrangian defined as $L=K-P$, which is the difference between the kinetic energy $\mathrm{K}$ and the potential energy $P$ of the form:

$$
K=\frac{1}{2} \dot{q}^{T} D(q) \dot{q}, P=P(q)
$$

with $D(q) \in R^{n \times n}$ being the symmetric and positive definite inertia matrix.

Robotic manipulator model. Letting $d_{i j}$ be the $i j$ th element of $D(q)$ and $\phi(q)=\frac{\partial P(q)}{\partial q}$ representing the gravitational effects, and substituting (2.2) into (2.1), we obtain the manipulator dynamic equation as follow:

$$
D(q) \ddot{q}+C(q, \dot{q}) \dot{q}+\phi(q)=u,
$$

where $C(q, \dot{q}) \in R^{n \times n}$ is a matrix representing the Coriolis and centrifugal effects, and the $k j$ th element of $C(q, \dot{q})$ is

$$
c_{k j}=\sum_{i=1}^{n} \frac{1}{2}\left(\frac{\partial d_{k j}}{\partial q_{i}}+\frac{\partial d_{k i}}{\partial q_{j}}+\frac{\partial d_{i j}}{\partial q_{k}}\right) \dot{q}_{i}
$$

Properties of the manipulator model. There are three important properties of the manipulator dynamics equation (2.3):

Property 2.1: The inertia matrix $D(q)$ is positive definite.

Property 2.2: The manipulator dynamic equation can be linearly parameterized, written as

$$
D(q) \ddot{q}+C(q, \dot{q}) \dot{q}+\phi(q)=Y(q, \dot{q}, \ddot{q}) p
$$

where $Y \in R^{n \times m}$ is the nonlinear regressor matrix and $p \in R^{m}$ is the $m$-dimensional 
parameter vector. This parameterization can be different since the choice of the parameter vector $p$ is not unique.

Property 3: The matrices $D(q)$ and $C(q, \dot{q})$ are not independent:

$$
x^{T}(M(q, \dot{q})-2 C(q, \dot{q})) x=0, \forall x \in R^{n},
$$

where $M(q, \dot{q})=\frac{d D(q)}{d t}$ whose ijth element is $\left(\frac{\partial d_{i j}}{\partial q}\right)^{T} \dot{q}$.

Adaptive control objective. Our objective is that given a bounded desired trajectory $q_{d}(t)$ along with its bounded derivatives $\dot{q}_{d}(t)$ and $\ddot{q}_{d}(t)$, design a feedback control input signal $u(t)$ for the manipulator system (2.3) without using the knowledge of the system parameters in $D(q), C(q, \dot{q})$ and $\phi(q)$, so that all signals in the closedloop system are bounded and the joint position $q(t)$ tracks $q_{d}(t)$ asymptotically.

If we choose a specific control strategy for this manipulator dynamic model:

$$
\tau=D(q)\left(\ddot{q}_{d}-K_{v} \dot{e}-K_{p} e\right)+C(q, \dot{q}) \dot{q}+\phi(q),
$$

which would result in

$$
\ddot{e}+K_{v} \dot{e}+K_{p} e=0,
$$

where $e=q-q_{d}, \dot{e}=\dot{q}-\dot{q}_{d}$, and $K_{p}$ and $K_{v}$ are positive definite diagonal gain matrices. This control strategy is usually referred to as the computed torque control. The error equation in (2.8) are globally stable, and desired performance can be achieved by appropriate choices of $K_{p}$ and $K_{v}$. However, the model used in the above control law is based on a static model of the manipulator, and hence if the load changes, the error equation is no longer homogeneous.

Some related preliminaries. We present some basic signal spaces and system 
operation definitions, and some key signal convergence properties for future use.

Definition 2.1. A vector signal $x(t) \in R^{n}$ is said to belong to the $L^{2}$ signal space, denoted as $x(t) \in L^{2}$, if $\int_{0}^{\infty}\left(x_{1}^{2}(t)+\cdots+x_{n}^{2}(t)\right) d t<\infty$.

Definition 2.2. A vector signal $x(t) \in R^{n}$ is said to belong to the $L^{\infty}$ signal space, denoted as $x(t) \in L^{\infty}$, if $\sup _{t \geq 0} \max _{1 \leq i \leq n}\left|x_{i}(t)\right|<\infty$.

It follows that $x(t) \in L^{2}$ or $x(t) \in \infty$ if and only if $x_{i}(t) \in L^{2}$ or $x_{i}(t) \in L^{\infty}$, $i=1,2, \ldots, n$.

To represent the dynamic system operations, for a stable transfer function $H(s)$, $z(t)=H(s)[w](t)$ or $z=H(s)[w]$ denotes the output $z(t)$ of $H(s)$ with input $w(t)$.

Lemma 2.1. If $e(t) \in L^{2}$ and $\dot{e}(t) \in L^{\infty}$, then $\lim _{t \rightarrow \infty} e(t)=0$.

Lemma 2.2. For a stable strictly proper transfer function $H(s)$, if $w(t) \in L^{2}$, then $z(t)=H(s)[w](t) \in L^{2}, \dot{z}(t) \in L^{2}, z(t) \in L^{\infty}$, and $\lim _{t \rightarrow \infty} z(t)=0$.

We assume that a manipulator is executing pick-and-place type tasks with load variations, during which major uncertainties would arise. That is, when the load changes, the parameters of the robot manipulator would change a lot due to the change of inertial properties and its load. This makes adaptive control a useful approach in this situation.

Adaptive control is a control methodology used by a controller which must adapt to a control system with parameters which vary, or are initially uncertain. There are two different approaches of adaptive control - direct and indirect adaptive control, depending on the signal they use to generate adaptive laws. Global stability of both approaches under certain assumptions has been proved by various researchers. 


\subsection{Direct Adaptive Control Design}

In direct adaptive control, we will discuss two typical algorithms, one of which requires the acceleration information, and the other does not. Notice that the adaptive laws used in direct adaptive control of robotic manipulators are driven by the tracking error $e(t)$.

\subsubsection{Design with Knowledge of the Acceleration $\ddot{q}(t)$}

With knowledge of the acceleration vector $\ddot{q}$, this algorithm, proposed by Craig et al. in 1987 [10], is based on the state feedback linearization concept and requires inversion of the estimated inertia matrix.

Control input signal. The control input signal is given as

$$
u=\hat{D}(q) v+\hat{C}(q, \dot{q}) \dot{q}+\hat{\phi}(q)
$$

where $v=\ddot{q}_{d}-K_{v} \dot{e}-K_{p} e, \hat{D}, \hat{C}$ and $\hat{\phi}$ are the estimates of $D, C$ and $\phi$ in (2.3) respectively.

Error equation. Substituting the control signal (2.9) into the manipulator dynamic equation (2.3) will result in an error equation:

$$
\hat{D}(q)\left(\ddot{e}+K_{v} \dot{e}+K_{p} e\right)=\tilde{D}(q) \ddot{q}+\tilde{C}(q, \dot{q}) \dot{q}+\tilde{\phi}=Y(q, \dot{q}, \ddot{q}) \tilde{p}
$$

where $\tilde{p}=p-\hat{p}$ is the parameter error vector, with $\hat{p}$ being the parameter estimate vector, and $\tilde{D}=D-\hat{D}, \tilde{C}=C-\hat{C}, \tilde{\phi}=\phi-\hat{\phi}$. 
Assuming $\hat{D}$ is positive definite, we may obtain

$$
\ddot{e}+K_{v} \dot{e}+K_{p} e=\underbrace{\hat{D}^{-1} Y(q, \dot{q}, \ddot{q})}_{\Phi} \tilde{p}=\Phi \tilde{p} .
$$

Express the error equation in state vector form for a clear expression as

$$
\dot{x}=\underbrace{\left[\begin{array}{cc}
0 & I \\
-K_{P} & -K_{v}
\end{array}\right]}_{A} x+\underbrace{\left[\begin{array}{c}
0 \\
I
\end{array}\right]}_{B} \Phi \tilde{p},
$$

with $x=[e, \dot{e}]^{T}$, and $\Phi=\hat{D}(q)^{-1} Y(q, \dot{q}, \ddot{q})$.

Adaptive law. Design the adaptive law as

$$
\dot{\tilde{p}}=-\Gamma^{-1} \Phi^{T} B^{T} P x
$$

where $\Gamma$ and $P$ are positive definite matrices and $P$ is the solution of the Lyapunov equation $A^{T} P+P A+Q=0$. This assures the global convergence of the error vector $x$, which will be shown in the stability analysis.

Stability analysis. The global stability of this algorithm can be proved as follows.

Theorem 2.1. The adaptive control scheme (2.9) - (2.13) guarantees that all the closed-loop signals are bounded, and $\lim _{t \rightarrow \infty} e(t)=0$.

Proof: This theorem holds because the positive definition function

$$
V=x^{T} P x+\tilde{p}^{T} \Gamma \tilde{p}
$$


has the property

$$
\dot{V}=-x^{T} Q x \leq 0
$$

from which we get that

$$
e(t) \in L^{\infty}, e(t) \in L^{2}, \dot{e} \in L^{\infty}, \tilde{p} \in L^{\infty}
$$

Therefore, the adaptive scheme is stable.

\subsubsection{Design without Knowledge of the Acceleration $\ddot{q}(t)$}

Slotine and Li developed an adaptive controller which can achieve the asymptotic tracking of a given trajectory $q_{d}(t)$ by the robot joint vector $q(t)$ without using the knowledge of the acceleration vector $\ddot{q}(t)$ [34]. This algorithm employs a variable transformation to convert the second-order dynamic system to a first-order error dynamic system in terms of a new error signal. The stability analysis of this algorithm is based on the property (2.6).

Control input signal. Using the variable transformation proposed in [34], we choose $K_{D}$ as an $n \times n$ constant matrix whose eigenvalues all have positive real parts, and define

$$
\ddot{q}_{r}=\ddot{q}_{d}+K_{D} \dot{e}, \dot{q}_{r}=\dot{q}_{d}+K_{D} e, r=\dot{e}+K_{D} e
$$

Then the control input signal is chosen as

$$
u=\hat{D}(q) \ddot{q}_{r}+\hat{C}(q, \dot{q}) \dot{q}_{r}+\hat{\phi}(q)+K_{v} r
$$

where $K_{v}$ is positive definite gain matrix.

Error equation. After the variable transformation and substituting the equation 
(2.18) into the manipulator dynamic equation (2.3), we get a new equation as

$$
D(q) \dot{r}+C(q, \dot{q}) r+K_{v} r=\tilde{D}(q) \ddot{q}_{r}+\tilde{C}(q, \dot{q}) \dot{q}_{r}+\tilde{\phi}
$$

where $\ddot{q}=\ddot{q}_{r}-\dot{r}$ and $\dot{q}=\dot{q}_{r}-r$.

Parameterizing (2.19), an error equation is obtained:

$$
D(q) \dot{r}+C(q, \dot{q}) r+K_{v} r=Y\left(q, \dot{q}, \ddot{q}_{r}, \dot{q}_{r}\right) \tilde{p}
$$

where $\tilde{p}=p-\hat{p}$.

Adaptive law. Design the adaptive law as

$$
\dot{\tilde{p}}=-\Gamma^{-1} Y^{T}\left(q, \dot{q}, \ddot{q}_{r}, \dot{q}_{r}\right) r
$$

with $\Gamma \in R^{r \times r}$ being an adaptation gain matrix such that $\Gamma=\Gamma^{T}>0$.

Stability analysis. The global stability of this algorithm can be proved as follows.

Theorem 2.2. The adaptive control scheme (2.18) - (2.21) guarantees that all the closed-loop signals are bounded, and $\lim _{t \rightarrow \infty} e(t)=0$.

Proof: This theorem is true because the positive definition function

$$
V=\frac{1}{2}\left(r^{T} D r+\tilde{p}^{T} \Gamma \tilde{p}\right),
$$

in which $D=D(q(t))$, has the property:

$$
\dot{V}=-r^{T} K_{v} r \leq 0
$$


from which we conclude that

$$
r(t) \in L^{\infty}, r(t) \in L^{2}, \tilde{p} \in L^{\infty}
$$

and, from $r=\dot{e}+K_{D} e$, that

$$
e(t) \in L^{\infty}, e(t) \in L^{2}, \dot{e}(t) \in L^{\infty}
$$

Hence we have $\lim _{t \rightarrow \infty} e(t)=0$, and from (2.17) (2.18), $u$ and $\dot{r}(t)$ are bounded, so that $\lim _{t \rightarrow \infty} r(t)=0$ and in turn $\lim _{t \rightarrow \infty} \dot{e}(t)=0$. Therefore, this theorem holds. $\nabla$

\subsection{Indirect Adaptive Control Design}

For indirect adaptive control design, the identification part and control part are separated. First, an identification model is used for error prediction of the manipulator, then the parameters will be estimated and the joint torque vector will be generated. What is different from the direct adaptive control is that adaptive law is driven by the torque prediction error, instead of the tracking error.

\subsubsection{Design with Knowledge of the Acceleration $\ddot{q}(t)$}

If it is assumed that the acceleration information is available, an identification model can be simply established using the position, velocity and acceleration information as follows. The prediction error, obtained from the the difference between the system output and the identification model, will be used for designing the adaptive law. 
Identification model. The identification model is built up as

$$
\hat{\tau}=\hat{D}(q) \ddot{q}+\hat{C}(q, \dot{q}) \dot{q}+\hat{\phi}(q)=Y(q, \dot{q}, \ddot{q}) \hat{p} .
$$

This identification model can be used in parallel with the plant dynamics to generate the torque prediction error.

Prediction error equation. The prediction error can be generated as

$$
\tilde{\tau}=u-\hat{\tau}=Y(q, \dot{q}, \ddot{q}) p-Y(q, \dot{q}, \ddot{q}) \hat{p}=Y(q, \dot{q}, \ddot{q}) \tilde{p},
$$

where $u$ is the actual torque input of the manipulator.

Adaptive law. Choose the adaptive law as

$$
\dot{\tilde{p}}=-\Gamma Y^{T}(q, \dot{q}, \ddot{q}) \tilde{\tau}
$$

with $\Gamma$ being a symmetric positive definite matrix.

Control input signal. The control input signal can be expressed as

$$
u=\hat{D}(q) v+\hat{C}(q, \dot{q}) \dot{q}+\hat{\phi}(q) .
$$

Stability analysis. The global stability of this algorithm can be proved as follows.

Theorem 2.3. The adaptive control scheme (2.26) - (2.29) guarantees that all the closed-loop signals are bounded, and $\lim _{t \rightarrow \infty} e(t)=0$. 
Proof: To prove this theorem, consider the positive definition function

$$
V=\frac{1}{2} \tilde{p}^{T} \Gamma^{-1} \tilde{p}
$$

which has

$$
\dot{V}=-\tilde{\tau}^{T} \tilde{\tau} \leq 0
$$

from which we conclude that

$$
\tilde{p} \in L^{\infty}, \tilde{\tau} \in L^{2}
$$

Since we have the prediction error equation as $\tilde{\tau}=Y(q, \dot{q}, \ddot{q}) \tilde{p}$, resulting to the conclusion that $\tilde{\tau} \in L^{\infty}$, and from $(2.26)$ we can obtain

$$
\tilde{\tau}=\hat{D}(q)\left(\ddot{e}+K_{v} \dot{e}+K_{p} e\right)
$$

which means

$$
e(t)=\left(s^{2} I+K_{v} s+K_{p}\right) \hat{D}^{-1}(q) \tilde{\tau}
$$

Recalling that $e=q_{d}-q$ and $q_{d}, \dot{q}_{d}$ is bounded, we obtain the desired property:

$$
e(t), \dot{e}(t) \in L^{2}, e(t) \in L^{\infty}
$$

So the theorem holds.

$\nabla$

\subsubsection{Design without Knowledge of the Acceleration $\ddot{q}(t)$}

Identification model. The identification model is chosen as

$$
\hat{\tau}=\hat{D}(q) \ddot{q}+\hat{C}(q, \dot{q}) \dot{q}+\hat{\phi}(q)=Y(q, \dot{q}, \ddot{q}) \hat{p}
$$


To generate an indirect adaptive control algorithm without using acceleration information, a method is proposed by Middleton and Goodwin (1988) that the identification model can be filtered by a first-order strictly proper filter $H(s)=\frac{\alpha}{s+\alpha}$, with $\alpha>0$. That is, for a term of the form $\ddot{\theta}_{2} f\left(\theta_{1}\right)$ in the regressor matrix $Y(q, \dot{q}, \ddot{q})$, it is true that

$$
H(s)\left\{\ddot{\theta}_{2} f\left(\theta_{1}\right)\right\}=s H(s)\left\{\dot{\theta}_{2} f\left(\theta_{1}\right)\right\}-H(s)\left\{\dot{\theta}_{1} \dot{\theta}_{2} \frac{\partial f\left(\theta_{1}\right)}{\partial \theta_{1}}\right\}
$$

from which a filtered equation can be generated as

$$
\hat{\tau}_{f}=H(s) \hat{\tau}=\underbrace{H(s) Y(q, \dot{q}, \ddot{q})}_{Y_{f}} \hat{p}=Y_{f}(q, \dot{q}) \hat{p}
$$

where the filtered regressor matrix $Y_{f}(q, \dot{q})$ contains only position and velocity measurements, and the acceleration signal $\ddot{q}$ is eliminated by filter $H(s)$.

Prediction error equation. The prediction error is defined as

$$
e_{I}=\tau_{f}-\hat{\tau}_{f}=Y_{f}(q, \dot{q}) \tilde{p}
$$

where $\tilde{p}=p-\hat{p}$, and $\tau_{f}=H(s) u$ is the actual filtered torque.

Adaptive law. The adaptive law is then chosen as

$$
\dot{\tilde{p}}=-\Gamma Y_{f}^{T}(q, \dot{q}) e_{I}
$$

with a symmetric positive definite matrix $\Gamma$. 
Control input signal. Generate the torque vector as

$$
u=\hat{D}(q)\left(\ddot{q}_{d}+K_{v} \dot{e}+K_{p} e\right)+\hat{C}(q, \dot{q}) \dot{q}+\hat{\phi}-\frac{1}{\alpha} Y_{f} \dot{\tilde{p}}-\frac{\hat{D}(q)}{\alpha}\left[\frac{d}{d t}\left(\hat{D}^{-1}(q)\right)\right] e_{I}
$$

The last two terms are included to deal with terms which arise in the adaptive case due to the commuting of time varying operators.

Stability analysis. The global stability of this algorithm can be proved as follows.

Theorem 2.4. The adaptive control scheme (2.36), (2.38) - (2.41) guarantees that all the closed-loop signals are bounded, and $\lim _{t \rightarrow \infty} e(t)=0$.

Proof: To prove this theorem, consider the positive definition function

$$
V=\frac{1}{2} \tilde{p}^{T} \Gamma^{-1} \tilde{p}
$$

which has

$$
\dot{V}=-e_{I}^{T} e_{I} \leq 0
$$

from which we conclude that

$$
\tilde{p} \in L^{\infty}, e_{I} \in L^{2}
$$

Since we have

$$
\begin{aligned}
H^{-1}(s) e_{I} & =\frac{s+\alpha}{\alpha} e_{I}=e_{I}+\frac{1}{\alpha} \dot{e}_{I}=Y_{f} \tilde{p}+\frac{1}{\alpha} \dot{Y}_{f} \tilde{p}+\frac{1}{\alpha} Y_{f} \dot{\tilde{p}} \\
& =\frac{s+\alpha}{\alpha} Y_{f} \tilde{p}+\frac{1}{\alpha} Y_{f} \dot{\tilde{p}}=Y \tilde{p}+\frac{1}{\alpha} Y_{f} \dot{\tilde{p}} \\
& =u-\hat{\tau}+\frac{1}{\alpha} Y_{f} \dot{\tilde{p}},
\end{aligned}
$$

and if the identification model (2.35) and torque vector (2.40) are substituted into 
equation (2.44), we get

$$
\begin{aligned}
H^{-1}(s) e_{I}= & e_{I}+\frac{1}{\alpha} \dot{e}_{I} \\
= & \hat{D}(q)\left(\ddot{q}_{d}+K_{v} \dot{e}+K_{p} e\right)+\hat{C}(q, \dot{q}) \dot{q}+\hat{\phi}-\frac{1}{\alpha} Y_{f} \dot{\tilde{p}}-\frac{\hat{D}(q)}{\alpha}\left[\frac{d}{d t}\left(\hat{D}^{-1}(q)\right)\right] e_{I} \\
& -\hat{D}(q) \ddot{q}-\hat{C}(q, \dot{q}) \dot{q}-\hat{\phi}+\frac{1}{\alpha} Y_{f} \dot{\tilde{p}} \\
= & \hat{D}(q)\left(\ddot{e}+K_{v} \dot{e}+K_{p} e\right)-\frac{\hat{D}(q)}{\alpha}\left[\frac{d}{d t}\left(\hat{D}^{-1}(q)\right)\right] e_{I}
\end{aligned}
$$

Thus we have

$$
\begin{aligned}
\hat{D}(q) H^{-1}(s)\left[\hat{D}^{-1}(q) e_{I}\right] & =\hat{D}(q)\left\{\hat{D}^{-1}(q) e_{I}+\frac{s}{\alpha}\left[\hat{D}^{-1}(q) e_{I}\right]\right\} \\
& =e_{I}+\frac{1}{\alpha} \dot{e}_{I}+\frac{\hat{D}(q)}{\alpha}\left[\frac{d}{d t}\left(\hat{D}^{-1}(q)\right)\right] e_{I} \\
& =\hat{D}(q)\left(\ddot{e}+K_{v} \dot{e}+K_{p} e\right),
\end{aligned}
$$

from which the error equation can be obtained as

$$
H^{-1}(s)\left[\hat{D}^{-1}(q) e_{I}\right]=\ddot{e}+K_{v} \dot{e}+K_{p} e
$$

Therefore, we get

$$
e(t)=\left(s^{2} I+K_{v} s+K_{p}\right)^{-1}(s+a)\left[\hat{D}^{-1}(q) e_{I}\right](t) .
$$

Since $\hat{D}^{-1}(q)$ is assumed to be nonsingular and we have already got $e_{I} \in L_{2}$, we can conclude that $e \rightarrow 0$ as $t \rightarrow \infty$. So the theorem holds. 


\subsection{Multiple-Model Control}

Multiple-model control is a general methodology for the design of adaptive control systems which can learn to operate efficiently in dynamical environments possessing a high degree of uncertainty. Multiple models are used to describe the different environments and the control is effected by switching to an appropriate controller followed by tuning or adaptation. This methodology is based on the use of multiple models, switching and tuning. In this section, we present the basic concepts involved in multiple-model control, and demonstrate how this problem can be posed mathematically. For better understanding, a multiple-model design based on adaptive robot control, which is proposed by Ciliz and Narendra in 1996 [7], will be included.

\subsubsection{General Methodology}

First, we introduce basic definitions and concepts about multiple-model control. Then the structure of the overall system and its operation will be briefly described.

System model. A model is considered as the representation of the essential parts of a system in a convenient form [24]. A model may have different forms depending on its purpose. Mathematical models are often used here because of their usefulness of representing the behavior of a system.

Environment. The behavior (or the input-output characteristics) of a system may change in different environments. Assume a system described by a set of differential equations:

$$
\begin{aligned}
\dot{x}(t) & =f[x(t), u(t), p], \\
y(t) & =h[x(t), p] .
\end{aligned}
$$


Obviously, the equations contain both the control plant and the external environments. If functions $f$ and $h$ are assumed to be fixed, different environments can be expressed by different values of the constant parameter vector $p$. Hence, different kinds of system uncertainties, such as variations of parameters, sensor and actuator failures, and external disturbances, can be considered as different environments.

Multiple models. A complex system is required to operate in different environments. When the environment changes, the input-output characteristics of the system will probably change rapidly. For a single-model control system, it has to adapt itself to the new environment before an appropriate control action can be taken. Such adaptation may be possible, but a large transient error may happen because of the slowness of adaptation process. Therefore, multiple-model control is used to identify the different environments as well as to control them rapidly. In some environments, different models may be available whose accuracy depends on the region in the state space where the system trajectories lie. Hence, multiple models may be preferable to a single model in many different situations.

Parametrization of models and controllers. To obtain a precise definition of the control problem, we assume that the plant model and the identification models can be parametrized in the same form. If $S$ is a closed bounded set in a finite dimensional parameter space, we assume that the plant parameter vector $p$ and the model parameter vectors $\hat{p}_{i}$ belong to $S$. Hence, the plant parameter vector can assume an infinite number of values and the objective is to improve the performance using a finite number of models.

Corresponding to each model parameter vector $\hat{p}_{i}$ there exists a neighborhood $S_{i} \subset S$ (also called the $i$ th environment), which has following properties: for all $p \in S_{i}$, the controller $C_{i}$ yields a tracking error which, according to some criterion 
function $J$, is smaller than a constant $\epsilon_{0}$. This $\epsilon$ can be considered as the maximum error that will using a fixed controller $C_{i}$, when $p \in S_{i}$.

If $p \in S_{i}$, the error criterion $J$ can be reduced by tuning the controller. To this end, the controller $C_{i}$ can be considered as providing initial condition for an adaptive controller. The objective of adaptation is to determine a controller $C_{i}^{*}$ such that the tracking error is smaller than a constant $\epsilon_{1} \ll \epsilon_{0}$. Since the plant parameter vector $p$ can lie anywhere in $S$, it is necessary to determine which controller $C_{i}$ must be used at every instant, and when switching should happen. Multiple identification models can be used at any instant in a control problem, but only one control input can be chosen. Hence, switching and tuning have to be carried out on the basis of identification errors rather than tracking errors.

Control system structure. In the structure of a multiple-model control system, there are $N$ identification models denoted by $\left\{I_{i}\right\}_{i=1}^{N}$ with corresponding outputs $\left\{y_{i}\right\}_{i=1}^{N}$. The identification errors $e_{i}=\hat{y}_{i}-y$ is measured at every instant by using a cost function $J_{i}(t), i=1,2, \ldots, N$, and the model corresponding to the smallest $J_{i}(t)$ is chosen to be the control input at that instant to the plant.

Adaptive and learning control. An anticipated environment is a subset $S_{i} \subset S$ which satisfies the above conditions, and the corresponding $\hat{p}_{i}$ is a known parameter vector. If $\bigcup_{i=1}^{N} S_{i}=S$, then every possible plant belongs to some anticipated environment. This corresponds to the case when learning concerning the plant is complete. In practice, this is rarely the case, and all the parameters $\hat{p}_{i}, \epsilon_{0}, \epsilon_{1}$ (and hence $S_{i}$ ) have to be learned on-line. These give rise to a host of theoretical questions, many of which are, as yet, unanswered.

The adaptive and learning control problem can be considered as a case where a finite number of models with parameter vectors $\left\{\hat{p}_{i}\right\}_{i=1}^{N}$, corresponding to $N$ antici- 
pated environments $\left\{S_{i}\right\}_{i=1}^{N}$, have already been learned. If at an instant the controller $C_{i}$ is used and the plant switches to an environment $S_{j} \neq S_{i}, i \leq j \leq N$, the performance index $J_{j}$ of the $j$ th model will be the minimum of the set $\left\{J_{i}\right\}_{i=1}^{N}$ and hence the controller will switch from $C_{i}$ to $C_{j}$. Adaptation of $\hat{p}_{j}$ then takes place within the set $S_{j}$. If, however, the new plant parameter vector $p \notin \bigcup_{i=1}^{N} S_{i}$, this corresponds to an unanticipated situation. In such a case, a new identification model $I_{N+1}$ (described by $\left.\hat{p}_{N+1}\right)$ and the corresponding environment $S_{N+1}$ have to be learned on-line. This is accomplished by continuing the adaptation process until the steady state error is smaller than $\epsilon_{1}$. Once $\hat{p}_{N+1}$ is determined. $S_{N+1}$ becomes an anticipated environment for all future performance of the system. As might be expected, the transient error while learning the appropriate control for unanticipated situations may be substantially greater than $\epsilon_{0}$.

Switching scheme. The function of the switching scheme is to monitor a performance index $J_{j}(t)$ based on the identification errors $e_{j}$ for each model $I_{j}$ and to switch to the controller corresponding to the model with the smallest value for $J_{j}(t)$. Our assumption in this strategy is that a small identification error leads to a small tracking error. The choice of the performance index is usually motivated by observations which can reliably estimate identifier accuracy. A general choice of performance index incorporating this feature has the form

$$
J_{j}(t)=\int_{0}^{t} e^{-\lambda(t-\tau)} e_{j}^{2}(\tau) d \tau
$$

Here $\lambda>0$ is free deign parameters, which determine the long-term memory of the index. The final control signal $u(t)$ will be chosen corresponding to the smallest value 
of the performance index, which can be expressed as

$$
u(t)=u_{(i)}(t), i=\arg _{k=1,2, \ldots, N} J_{k}(t)
$$

\subsubsection{Multiple-Model Design of Indirect Adaptive Control}

The indirect adaptive control design using filtered prediction model (introduced in 2.3.2), which was proposed by Ciliz and Narendra in 1996 [7], was further extended to a multiple-model adaptive control scheme. In this subsection, we will describe it as an example for multiple-model control design of robotic manipulators.

This adaptive controller architecture utilizes the same structural dynamic model with their single model control scheme, but with different initial estimates of the inertial parameters of the manipulator and its load. Denote $\mathrm{N}$ adaptive identification models by $I_{j}$ with $j=1, \ldots, N$, and corresponding controllers $C_{j}$, which use parameter estimates obtained from the identification models $I_{j}$. The main idea is to choose a model among $I_{j}$ that closely approximates the manipulator at that instant to generate the control vector $u$.

Prediction errors. The filtered plant model $\tau_{f}$ was used to construct a set of multiple prediction signals $\hat{\tau}_{f(i)}$ :

$$
\hat{\tau}_{f(i)}=Y_{f}(q, \dot{q}) \hat{p}_{(i)}
$$

where $\hat{p}_{(i)}$ are the multiple adaptive estimates of $p$, for $i=1,2, \ldots, N$, with $N$ being a positive integer of interest. Generate the prediction errors as

$$
\tilde{\tau}_{f(i)}=\tau_{f}-\hat{\tau}_{f(i)}=Y_{f}(q, \dot{q}) \tilde{p}_{(i)}
$$


where $\tilde{p}_{(i)}=p-\hat{p}_{(i)}$, and $\tau_{f}=H(s)[\tau]$.

Adaptive laws. The adaptive laws for updating the parameter estimates $\hat{p}_{(i)}(t)$ are chosen as

$$
\dot{\hat{p}}_{(i)}=-\Gamma_{(i)} Y_{f}^{T}(q, \dot{q}) \tilde{\tau}_{f(i)}, \Gamma_{(i)}=\Gamma_{(i)}^{T}>0, i=1,2, \ldots, N
$$

where $\hat{p}_{(i)}(0)$ are chosen from different locations in the parameter space $R^{m}, i=$ $1,2, \ldots, N$, one of them is closer to the true parameter vector $p$ than others.

Control input signals. With the prediction errors (2.54), adaptive laws (2.55) and different choices of $\hat{p}_{(i)}(0)$, multiple estimates $\hat{p}_{(i)}$ can be obtained, which are used to construct the multiple adaptive estimates $\hat{D}_{(i)}(q), \hat{C}_{i}(q, \dot{q})$ and $\hat{\phi}_{(i)}(q)$. Under the assumption that $\hat{D}_{(i)}(q(t))=\hat{D}_{(i)}(\hat{p}(t), q(t)$ are assumed to be nonsingular for all $t$, and $\frac{d}{d t}\left(\hat{D}_{(i)}^{-1}(\hat{p}(t), q(t))\right)$ are well-defined for all $i=1,2, \ldots, N$, the above signals are employed to generate a set of control input signals as

$$
\begin{aligned}
u_{(i)}= & \hat{D}_{(i)}(q)\left(\ddot{q}_{d}-K_{v(i)} \dot{e}-K_{p(i)} e\right)+\hat{C}_{(i)}(q, \dot{q}) \dot{q}+\hat{\phi}_{(i)}(q) \\
& -\frac{1}{\alpha} Y_{f} \dot{\tilde{p}}_{(i)}+\frac{\hat{D}_{(i)}(q)}{\alpha}\left[\frac{d}{d t}\left(\hat{D}_{(i)}^{-1}\right)\right] \tilde{\tau}_{f(i)},
\end{aligned}
$$

where $e(t)=q-q_{d}, \dot{e}=\dot{q}-\dot{q}_{d}$, and $K_{p(i)}$ and $K_{p(i)}$ are diagonal and positive definite gain matrices, $i=1,2, \ldots, N$.

Stability analysis. Each estimate $\hat{p}_{(i)}$ and corresponding prediction error $\tilde{\tau}_{f(i)}=$ $\tau_{f}-\hat{\tau}_{f(i)}$ can guarantee the desired properties: $\hat{p}_{(i)} \in L^{\infty}, \tilde{\tau}_{f(i)} \in L^{2}, i=1,2, \ldots, N$. And if $\tau(t)=\tau_{(i)}(t), t \geq 0$, for any chosen $i$, the closed-loop system is stable and $\lim _{t \rightarrow \infty} e(t)=0$. 
Proof: For each identification model, there is a Lyapunov function

$$
V_{(i)}=\frac{1}{2} \tilde{p}_{(i)}^{T} \Gamma^{-1} \tilde{p}_{(i)}
$$

which has a property that

$$
\dot{V}_{(i)}=-\tilde{\tau}_{f(i)}^{T} \tilde{\tau}_{f(i)}
$$

So they concluded that $\tilde{\tau}_{f(i)} \in L_{2}$ and $\tilde{p}_{(i)} \in L_{\infty}$ for all $i=1, \ldots, N$.

The control input (2.55) results in the following error equation:

$$
H^{-1}(s) \hat{D}_{(i)}^{-1}(q) \tilde{\tau}_{f(i)}=\ddot{e}+K_{v} \dot{e}+K_{p} e
$$

Since $\hat{D}_{(i)}^{-1} \in L_{\infty}$, then $\hat{D}_{(i)}^{-1} \tilde{\tau}_{f(i)} \in L^{2}$, so that $e, \dot{e} \rightarrow 0$ as $t \rightarrow \infty$ with all signals remaining bounded, and $\tilde{\tau}_{f(i)} \in L^{2} \cap L^{\infty}$. Also, from (2.55), (2.58) and $\ddot{q} \in L^{\infty}$, it can be obtained that $\dot{\tilde{\tau}}_{f(i)} \in L^{\infty}$, since $Y_{f}, \tilde{p}_{(i)}, \dot{Y}_{f}, \dot{\tilde{p}}_{(i)} \in L_{\infty}$. Hence, $\tilde{\tau}_{f(i)} \rightarrow 0$ as $t \rightarrow \infty$ since it is bounded and uniformly continuous. Therefore, desired properties can be proved.

Performance indexes. Due to the uncertainty of $\hat{p}_{(i)}(0)$ relative to the true parameter vector $p \in R^{m}$, a single-model adaptive control scheme may not provide a satisfying transient response. To improve the tracking performance, Ciliz and Narendra proposed a switching rule to choose an appropriate one among the set of controllers. Their selection criterion is to choose a controller with the smallest prediction error $\tilde{\tau}_{f(i)}(t)=\tau_{f}(t)-\hat{\tau}_{f(i)}(t)$, since a smaller error would yield a better performance. The quadratic performance index they chose is:

$$
J_{(i)}(t)=\gamma \tilde{\tau}_{f(i)}^{T}(t) \tilde{\tau}_{f(i)}(t)+\beta \int_{0}^{t} \tilde{\tau}_{f(i)}^{T}(\zeta) \tilde{\tau}_{f(i)}(\zeta) d \zeta
$$


where $\gamma>0$ and $\beta>0$ are weights that can be tuned experimentally by monitoring the tracking performance. Hence if they know there would be sudden dynamical changes in the robot system, the parameter $\gamma$ could be increased to generate an abrupt change in $\tilde{\tau}_{f(i)}(t)$ which could be identified easily. However, increasing $\gamma$ may not be sufficient to detect a sudden change in $\tilde{\tau}_{f(i)}(t)$ if the integral term dominates $J_{(i)}(t)$ substantially in some cases, so a modified performance criterion is given as

$$
J_{(i)}(t)=\gamma \tilde{\tau}_{f(i)}^{T}(t) \tilde{\tau}_{f(i)}(t)+\beta \int_{t-T}^{T} \tilde{\tau}_{f(i)}^{T}(\zeta) \tilde{\tau}_{f(i)}(\zeta) d \zeta
$$

where $T$ can be experimentally chosen. With this modification, the integral of the error square would be computed over a sliding window, so that capturing the longterm error effects (depending on the length of $T$ ) makes it possible to detect the transient peaks in $\tilde{\tau}_{f(i)}^{T}(t) \tilde{\tau}_{f(i)}(t)$.

Based on this performance indexes, the final control signal $\tau(t)$ is chosen as

$$
\tau(t)=\tau_{(i)}(t), i=\arg \min _{k=1,2, \ldots, N} J_{(k)}(t),
$$

which means that $\tau(t)$ is set to be $\tau_{(i)}(t)$ with $J_{(i)}(t)$ being the minimum of $J_{(k)}(t)$ for all $k=1,2, \ldots, N$.

\subsection{Problem Statement}

After understanding those existing algorithms, we found that the main drawbacks of these algorithms are:

Some of them requires the information of acceleration vector, which is difficult to measure, and may bring large errors to the control system in practical issues; 
or, some of them do not have the basic structure for development to multiple-model control scheme, which makes it impossible for performance improvement;

or, some of them have complex compensation terms in the control input signal, leading to difficulties in computing and analyzing system parameters;

and none of them provides a way to guarantee the crucial property for stability analysis, which is that the estimate of inertia matrix is invertible.

In this thesis, our aim is to propose a new multiple-model adaptive control scheme based on system prediction for robotic manipulators. This scheme is derived from Slotine's algorithm, since the variable transformation method will also be used to eliminate the requirements of acceleration vector. However, we plan to use a different control design to solve or avoid all the problems mentioned above.

Thesis goals. The specific control objective can be defined as follows. This thesis is dedicated to design a multiple-model adaptive control scheme, which has the potential to choose an appropriate control signal $u(t)$ for the robotic manipulator system (2.3) with unknown system parameters in matrices $D(q), C(q, \dot{q})$ and $\phi(q)$ to achieve trajectory tracking. That means, under this control scheme, all the signals in the closed loop system are bounded, the joint position $q(t)$ tracks the desired joint position $q_{d}(t)$ asymptotically, and the transient performance is improved. We will approach this goal step by step. 


\section{Chapter 3}

\section{A Dynamic Prediction Error Based Adaptive Control Scheme}

Since the algorithms introduced in Chapter 2 have some drawbacks, in this chapter, we design a new dynamic prediction error based adaptive control scheme, whose parameter estimation scheme is different from those estimator-based adaptive control algorithms in the literature. This adaptive control scheme contains the needed basic structure for developing multiple-model adaptive control design, and can avoid drawbacks mentioned above. After understanding this single-model adaptive control scheme, it will be used as the baseline control scheme for our multiple-model design in next chapter.

\subsection{Nominal Controller}

A nominal controller is a controller designed for a system whose parameters are known. Nominal controllers are used for the design of adaptive controllers. 
Using variable transformation as

$$
v=\dot{q}_{d}-\Lambda\left(q-q_{d}\right), u s=\dot{q}-v, e=q-q_{d}
$$

where $\Lambda$ is chosen as an $n \times n$ constant matrix whose eigenvalues all have positive real parts. Substituting equation (3.1) into (2.3) and we obtain

$$
D(q) \dot{s}+C(q, \dot{q}) s=u-D(q) \dot{v}-C(q, \dot{q}) v-\phi(q)
$$

In this expression, $s=\dot{e}+\Lambda e, \dot{s}=\ddot{q}-\dot{v}$, and $s, v, \dot{v}$ depend only on $q, q_{d}, \dot{q}, \dot{q}_{d}, \ddot{q}_{d}$ and not on the joint acceleration vector $\ddot{q}(t)$. The term of $\dot{s}=\ddot{q}-\dot{v}$ is related to the joint acceleration vector, but it is not needed in our control design.

Then we introduce the parametrization as

$$
D(q) \dot{v}+C(q, \dot{q}) v+\phi(q)=Y\left(q, q_{d}, \dot{q}, \dot{q}_{d}, \ddot{q}_{d}\right) \theta^{*}
$$

where $\theta^{*} \in R^{m}$ is a parameter vector containing all unknown parameters in the manipulator system for some $m>0$, and $Y\left(q, q_{d}, \dot{q}, \dot{q}_{d}, \ddot{q}_{d}\right)$ is an $n \times m$ regressor matrix of known functions. $Y\left(q, q_{d}, \dot{q}, \dot{q}_{d}, \ddot{q}_{d}\right)$ is bounded for all bounded signals $q, q_{d}, \dot{q}, \dot{q}_{d}, \ddot{q}_{d}$.

After the variable transformation (3.1) and parametrization (3.3), an parametrized system model without requirement of the joint acceleration vector $\ddot{q}(t)$ is presented as

$$
D(q) \dot{s}+C(q, \dot{q}) s=u-Y\left(q, q_{d}, \dot{q}, \dot{q}_{d}, \ddot{q}_{d}\right) \theta^{*}
$$

Control Design for known $\theta^{*}$. For this parametrized system model, a nominal 
controller may be designed with knowledge of the parameter vector $\theta^{*}$ :

$$
u(t)=C(q, \dot{q}) s(t)+Y\left(q, q_{d}, \dot{q}, \dot{q}_{d}, \ddot{q}_{d}\right) \theta^{*}-D(q) \Lambda_{1} s(t)
$$

where $\Lambda_{1}$ is an $n \times n$ constant matrix whose eigenvalues all have positive real parts. This controller would result in a desired closed-loop system as

$$
D(q)\left(\dot{s}(t)+\Lambda_{1} s(t)\right)=0
$$

which means $\dot{s}(t)+\Lambda_{1} s(t)=0$ since $D(q)$ is nonsingular. Consequently, $s(t)$ is bounded and $\lim _{t \rightarrow \infty} s(t)=0$ exponentially, so that all system signals are bounded and $\lim _{t \rightarrow \infty} e(t)=0$.

\subsection{Adaptive Parameter Estimation Design}

In the cases where the system parameters are unknown, an adaptive predictor for the error signal $s(t)$ is necessary to estimate the unknown parameters in $D(q), C(q, \dot{q})$, and $\theta^{*}$ to generate the adaptive version of the controller (3.5). An adaptive parameter adaptive law is also needed for generating and updating desired parameter estimates. Bounded parameter errors would be achieved with appropriate choice of the adaptive law. What different from other algorithms in the literature is we use parameter pro-

jection to guarantee that the matrix $\hat{D}(q)$ will remain positive definite and invertible during the adaptation process.

\subsubsection{Parameter Estimation}

The parameter estimation scheme consists of an adaptive predictor for the error signal and adaptive parameter adaptive laws. 
Adaptive predictor. In order to construct a parameter estimation scheme based on the system model (3.4), the first step is to introduce an adaptive dynamic predictor to generate a prediction signal $\hat{s}(t)$ for the error signal $s(t)$, so the system model $(3.4)$ becomes

$$
\hat{D}(q) \dot{\hat{s}}+\hat{C}(q, \dot{q}) \hat{s}=u-Y\left(q, q_{d}, \dot{q}, \dot{q}_{d}, \ddot{q}_{d}\right) \theta+K_{0} \tilde{s}
$$

where $\theta$ is an adaptive estimate of $\theta^{*}, \hat{D}(q)$ and $\hat{C}(q, \dot{q})$ are estimates of $D(q)$ and $C(q, \dot{q}), K_{0} \in R^{n \times n}$ is a design parameter matrix with the property that $K_{0}=K_{0}^{T}>0$, and $\tilde{s}(t)=s(t)-\hat{s}(t)$ is the dynamic prediction error (the signal $\hat{s}(t)$ may be called as a posteriori prediction of the signal $s(t)$, based on information available up to time $t$ when $s(t)$ is also available).

Use equation (3.4) and (3.7), we may get the prediction error equation

$$
D(q) \dot{\tilde{s}}+C(q, \dot{q}) \tilde{s}=\tilde{D}(q) \dot{\hat{s}}+\tilde{C}(q, \dot{q}) \hat{s}+Y\left(q, q_{d}, \dot{q}, \dot{q}_{d}, \ddot{q}_{d}\right)\left(\theta-\theta^{*}\right)-K_{0} \tilde{s}
$$

where

$$
\tilde{D}(q)=\hat{D}(q)-D(q), \tilde{C}(q, \dot{q})=\hat{C}(q, \dot{q})-C(q, \dot{q})
$$

Parametrization of $D(q) \dot{\hat{s}}$ and $C(q, \dot{q}) \hat{s}$. Next step is to properly parametrize $D(q) \dot{\hat{s}}$ and $C(q, \dot{q}) \hat{s}$ to estimate the parameters of $D(q)$ and $C(q, \dot{q})$ and generate stable estimates of $D(q), C(q, \dot{q})$ and an invertible estimate of $D(q)$. So we parametrize $C(q, \dot{q}) \hat{s}$ as

$$
C(q, \dot{q}) \hat{s}=Y_{C}(q, \dot{q}, \hat{s}) \theta_{C}^{*}
$$

for some known signal matrix $Y_{C}(q, \dot{q}, \hat{s}) \in R^{n \times n_{c}}$ and unknown parameter vector $\theta_{C}^{*} \in R^{n_{c}}$. Signals $\hat{s}(t)$ and $\dot{\hat{s}}$ are available from the predictor (3.7). 
The parametrization of $D(q) \dot{\hat{s}}(t)$ is more complicated. We first express $D(q)$ as

$$
D(q)=\left\{d_{i j}(q)\right\}, d_{i j}(q)=\theta_{i j}^{* T} \phi_{i j}(q)
$$

where $d_{i j}(q)$ is the $i j$ th element of $D(q), \theta_{i j}^{*} \in R^{m_{i j}}$ is some unknown parameter vector, and $\phi_{i j}(q) \in R^{m_{i j}}$ is some known signal vector, $i, j=1,, 2, \ldots, n$. Our goal is to have $\theta_{i j}^{*}$ appearing explicitly for all $i, j=1,2, \ldots, n$ after parametrization of $D(q) \dot{\hat{s}}$, so that the estimates $\theta_{i j}$ of $\theta_{i j}^{*}$ can be used to construct the estimate $\hat{D}(q)=\left\{\theta_{i j}^{T} \phi_{i j}(q)\right\}$ of $D(q)=\left\{\theta_{i j}^{* T} \phi_{i j}(q)\right\}$. This explicit $\hat{D}(q)$ is necessary to implement an adaptive version of $D(q) \Gamma_{1} s(t)$ in the nominal controller (3.5).

To this end, we introduce the following expressions for $\dot{\hat{s}}(t)=\left[\dot{\hat{\hat{s}}}_{1}(t), \dot{\hat{s}}_{2}(t), \ldots, \dot{\hat{s}}_{n}(t)\right]^{T}$ :

$$
\begin{aligned}
\theta_{i}^{*} & =\left[\theta_{i 1}^{* T}, \theta_{i 2}^{* T}, \ldots \theta_{i n}^{* T}\right]^{T}, i=1,2, \ldots, n \\
\theta_{D}^{*} & =\left[\theta_{1}^{* T}, \theta_{2}^{* T}, \ldots, \theta_{n}^{* T}\right]^{T} \\
\phi_{s i}(t) & =\left[\phi_{i 1}^{T}(t) \dot{\hat{s}}_{1}(t), \phi_{i 2}^{T}(t) \dot{\hat{s}}_{2}(t), \ldots, \phi_{i n}^{T}(t) \dot{\hat{s}}_{n}(t)\right]^{T} \\
Y_{D}(q, \dot{\hat{s}}) & =\operatorname{diag}\left\{\phi_{s 1}^{T}(t), \phi_{s 2}^{T}(t), \ldots, \phi_{s n}^{T}(t)\right\}
\end{aligned}
$$

and do parametrization of $D(q) \dot{\hat{s}}$ as

$$
D(q) \dot{\hat{s}}=Y_{D}(q, \dot{\hat{s}}) \theta_{D}^{*}
$$

where $Y_{D}(q, \dot{\hat{s}}) \in R^{n \times n_{D}}\left(n_{D}=\sum_{i=1}^{n} \sum_{j=1}^{n} m_{i j}\right)$ is a matrix of known parameters (whose off block diagonal elements are all zero) and $\theta_{D}^{*} \in R^{n_{D}}$ is a vector of unknown parameters. Thus, we would get an important property that the estimate $\hat{D}(q)$ of $D(q)$ can be directly obtained from the estimate $\theta_{D}(t)$ of $\theta_{D}^{*}$ as

$$
\hat{D}(q)=\hat{D}\left(q, \theta_{D}\right)=\left\{\hat{d}_{i j}\left(q, \theta_{i j}\right)\right\}, \hat{d}_{i j}\left(q, \theta_{i j}\right)=\theta_{i j}^{T} \phi_{i j}(q)
$$


where $\hat{d}_{i j}(q)$ are the $i j$ th element of $\hat{D}(q)$, and $\theta_{i j}$ are the estimates of $\theta_{i j}^{*}$, as the components of $\theta_{D}, i, j=1,2, \ldots, n$, corresponding to $\theta_{i j}^{*}$ in $\theta_{D}^{*}$.

With the parametrization scheme, we have

$$
D(q) \dot{\hat{s}}+C(q, \dot{q}) \hat{s}=Y_{D}(q, \dot{\hat{s}}) \theta_{D}^{*}+Y_{C}(q, \dot{q}, \hat{s}) \theta_{C}^{*}
$$

and the estimated version correspondingly,

$$
\hat{D}(q) \dot{\hat{s}}+\hat{C}(q, \dot{q}) \hat{s}=Y_{D}(q, \dot{\hat{s}}) \theta_{D}+Y_{C}(q, \dot{q}, \hat{s}) \theta_{C}
$$

where $\theta_{D}(t)$ and $\theta_{C}(t)$ are the estimates of $\theta_{D}^{*}$ and $\theta_{C}^{*}$. Subtract equation (3.16) from $(3.15)$ as

$$
\tilde{D}(q) \dot{\hat{s}}+\tilde{C}(q, \dot{q}) \hat{s}=Y_{D}(q, \dot{\hat{s}})\left(\theta_{D}-\theta_{D}^{*}\right)+Y_{C}(q, \dot{q}, \hat{s})\left(\theta_{C}-\theta_{C}^{*}\right)
$$

Then the error equation (3.8) becomes

$$
\begin{aligned}
D(q) \dot{\tilde{s}}+C(q, \dot{q}) \tilde{s}= & Y_{D}(q, \dot{\hat{s}})\left(\theta_{D}-\theta_{D}^{*}\right)+Y_{C}(q, \dot{q}, \hat{s})\left(\theta_{C}-\theta_{C}^{*}\right) \\
& +Y\left(q, q_{d}, \dot{q}, \dot{q}_{d}, \ddot{q}_{d}\right)\left(\theta-\theta^{*}\right)-K_{0} \tilde{s}
\end{aligned}
$$

Remark 3.1. Notice that we did parametrization not only in equation (3.3): $D(q) \dot{v}+$ $C(q, \dot{q}) v+\phi(q)=Y\left(q, q_{d}, \dot{q}, \dot{q}_{d}, \ddot{q}_{d}\right) \theta^{*}$, but also in equation $(3.10): C(q, \dot{q}) \hat{s}=Y_{C}(q, \dot{q}, \hat{s}) \theta_{C}^{*}$ and equation (3.13): $D(q) \dot{\hat{s}}=Y_{D}(q, \dot{\hat{s}}) \theta_{D}^{*}$. The reason of such a reparametrization is to reduce the total number of parameters to be estimated. Because for $\tilde{D}(q) \dot{\hat{s}}, \tilde{C}(q, \dot{q}) \hat{s}$ and $Y\left(q, q_{d}, \dot{q}, \dot{q}_{d}, \ddot{q}_{d}\right)\left(\theta-\theta^{*}\right)$ in the former error equation (3.8), the reparametrization allows us to combine the parameters of $D(q)$ in $D(q) \dot{\hat{s}}$ and those of $D(q)$ in $D(q) \dot{v}$ together, and similarly, allows us to combine the parameters of $C(q, \dot{q})$ in $C(q, \dot{q}) \hat{s}$ and 
those of $C(q, \dot{q})$ in $C(q, \dot{q}) v$ together. Thus, parameters in $\theta^{*}$ could be reduced and contain the parameters of $\phi(q)$ only, in this way we obtain a more efficient adaptive scheme.

Adaptive laws. Based on the error equation (3.18), we choose the adaptive laws for $\theta_{D}(t), \theta_{C}(t)$ and $\theta(t)$ as

$$
\begin{aligned}
\dot{\theta}_{D}(t) & =-\Gamma_{D} Y_{D}^{T}(q, \dot{\hat{s}}) \tilde{s}(t) \\
\dot{\theta}_{C}(t) & =-\Gamma_{C} Y_{C}^{T}(q, \dot{q}, \hat{s}) \tilde{s}(t) \\
\dot{\theta}(t) & =-\Gamma Y^{T}\left(q, q_{d}, \dot{q}, \dot{q}_{d}, \ddot{q}_{d}\right) \tilde{s}(t)
\end{aligned}
$$

where $\Gamma_{D} \in R^{n_{D} \times n_{D}}, \Gamma_{C} \in R^{n_{C} \times n_{C}}$ and $\Gamma \in R^{r \times r}$ are adaptive gain matrices chosen to satisfy $\Gamma_{D}=\Gamma_{D}^{T}>0, \Gamma_{C}=\Gamma_{C}^{T}>0$ and $\Gamma=\Gamma^{T}>0$.

These adaptive laws are chosen to guarantee the desired properties of this adaptive parameter estimation scheme. Detailed proof is shown as follows.

Lemma 3.1. The adaptive scheme consisting of (3.19)-(3.21) ensures that the prediction errors $\tilde{s}(t)=s(t)-\hat{s}(t)$, and the parameter errors $\theta_{D}(t)-\theta_{D}^{*}, \theta_{C}(t)-\theta_{C}^{*}$ and $\theta(t)-\theta^{*}$ are all bounded, and $\tilde{s}(t) \in L^{2}$.

Proof: Consider the positive definite function

$$
V\left(\tilde{s}, \tilde{\theta}_{D}, \tilde{\theta}_{C}, \tilde{\theta}\right)=\frac{1}{2}\left(\tilde{s}^{T} D \tilde{s}+\tilde{\theta}_{D}^{T} \Gamma_{D}^{-1} \tilde{\theta}_{D}+\tilde{\theta}_{C}^{T} \Gamma_{C}^{-1} \tilde{\theta}_{C}+\tilde{\theta}^{T} \Gamma^{-1} \tilde{\theta}\right)
$$

With the previous error equation (3.8) and the choices of adaptive laws (3.19)-(3.21), 
differentiating $V\left(\tilde{s}, \tilde{\theta}_{D}, \tilde{\theta}_{C}, \tilde{\theta}\right)$ yields

$$
\begin{aligned}
\dot{V} & =\tilde{s}^{T} D \dot{\tilde{s}}+\frac{1}{2} \tilde{s}^{T} \dot{D} \tilde{s}+\tilde{\theta}_{D}^{T} \Gamma_{D}^{-1} \dot{\tilde{\theta}}_{D}+\tilde{\theta}_{C}^{T} \Gamma_{C}^{-1} \dot{\tilde{\theta}}_{C}+\tilde{\theta}^{T} \Gamma^{-1} \dot{\tilde{\theta}} \\
& =\tilde{s}^{T}(D \dot{\tilde{s}}+C \tilde{s})-\left(\tilde{\theta}_{D}^{T} Y_{D}^{T}+\tilde{\theta}_{C}^{T} Y_{C}^{T}+\tilde{\theta}^{T} Y^{T}\right) \tilde{s} \\
& =\tilde{s}^{T}\left(Y_{D} \tilde{\theta}_{D}+Y_{C} \tilde{\theta}_{C}+Y \tilde{\theta}-K_{0} \tilde{s}\right)-\left(\tilde{\theta}_{D}^{T} Y_{D}^{T}+\tilde{\theta}_{C}^{T} Y_{C}^{T}+\tilde{\theta}^{T} Y^{T}\right) \tilde{s} \\
& =-\tilde{s}^{T}(t) K_{0} \tilde{s}(t) \leq 0 .
\end{aligned}
$$

Consequently we have

$$
\tilde{\theta}_{D}(t), \tilde{\theta}_{C}(t), \tilde{\theta}(t) \in L^{\infty}, \tilde{s}(t) \in L^{\infty} \cap L^{2}
$$

so that the lemma holds.

In the adaptive control scheme we present above, the parametrization of $D(q) \dot{\hat{s}}$ may be compacted using some property of $D(q)$, and further compaction may be made for some special cases.

\subsubsection{Compact Parametrization of $D(q) \dot{\hat{s}}$}

Since we know that $D(q)$ is a symmetric matrix, which means that $d_{i j}(q)=d_{j i}(q)$, or that $\theta_{i j}^{*}=\theta_{j i}^{*}$ and $\phi_{i j}(q)=\phi_{j i}(q)$ from equation (3.11), we can obtain a more compact adaptive control scheme for all $i, j$ by modifying the way of parametrization. 
kjConsider a 2-link planar manipulator, whose inertia matrix $D(q)$ is

$$
\begin{aligned}
d_{11}(q) & =M_{1} l_{c 1}^{2}+M_{2}\left(l_{1}^{2}+l_{c 2}^{2}+2 l_{1} l_{c 2} \cos \left(q_{2}\right)\right)+I_{1}+I_{2} \\
& =M_{1} l_{c 1}^{2}+M_{2}\left(l_{1}^{2}+l_{c 2}^{2}\right)+I_{1}+I_{2}+2 M_{2} l_{1} l_{c 2} \cos \left(q_{2}\right) \\
d_{12}(q) & =d_{21}(q)=M_{2}\left(l_{c 2}^{2}+l_{1} l_{c 1} \cos \left(q_{2}\right)\right)+I_{2} \\
& =M_{2} l_{c 2}^{2}+I_{2}+M_{2} l_{1} l_{c 2} \cos \left(q_{2}\right) \\
d_{22}(q) & =M_{2} l_{c 2}^{2}+I_{2} .
\end{aligned}
$$

The associated parameter vectors are

$$
\begin{aligned}
& \theta_{11}^{*}=\left[M_{1} l_{c 1}^{2}+M_{2}\left(l_{1}^{2}+l_{c 2}^{2}\right)+I_{1}+I_{2}, 2 M_{2} l_{1} l_{c 2}\right]^{T}, \phi_{11}(q)=\left[1, \cos \left(q_{2}\right)\right]^{T} \\
& \theta_{12}^{*}=\left[M_{2} l_{c 2}^{2}+I_{2}, M_{2} l_{1} l_{c 2}\right]^{T}, \phi_{11}(q)=\left[1, \cos \left(q_{2}\right)\right]^{T} \\
& \theta_{22}^{*}=M_{2} l_{c 2}^{2}+I_{2}, \phi_{22}(q)=1,
\end{aligned}
$$

which contains five parameters: $\theta_{111}^{*}, \theta_{112}^{*}, \theta_{121}^{*}, \theta_{122}^{*}, \theta_{22}^{*}$. However, this model can be expressed using only three parameters:

$$
\begin{aligned}
\theta_{D}^{*} & =\left[\theta_{D 1}^{*}, \theta_{D 2}^{*}, \theta_{D 3}^{*}\right]^{T} \\
& =\left[M_{1} l_{c 1}^{2}+M_{2}\left(l_{1}^{2}+l_{c 2}^{2}\right)+I_{1}+I_{2}, M_{2} l_{1} l_{c 2}, M_{2} l_{c 2}^{2}+I_{2}\right]^{T} .
\end{aligned}
$$

Thus, $d_{i j}(q)$ can be expressed as

$$
D(q) \dot{\hat{s}}(t)=\left[\begin{array}{ccc}
\dot{\hat{s}}_{1}(t) & \left(2 \dot{\hat{s}}_{1}(t)+\dot{\hat{s}}_{2}(t)\right) \cos \left(q_{2}\right) & \dot{\hat{s}}_{2}(t) \\
0 & \dot{\hat{s}}_{1}(t) \cos \left(q_{2}\right) & \dot{\hat{s}}_{1}(t)+\dot{\hat{s}}_{2}(t)
\end{array}\right]\left[\begin{array}{c}
\theta_{D 1}^{*} \\
\theta_{D 2}^{*} \\
\theta_{D 3}^{*}
\end{array}\right]
$$

We can see that the parametrization is further simplified, comparing with (3.36) and 
$(3.42)-(3.44)$

\subsubsection{A Parameter Projection Scheme}

In the analysis of nominal closed-loop control system (3.6): $D(q)\left(\dot{s}(t)+\Lambda_{1} s(t)\right)=$ 0 , where $-\Lambda_{1}$ is an $n \times n$ constant and stable matrix, we obtain the desired properties based on the condition that $D(q)$ is nonsingular. In the predictor-based adaptive version in Section 3.2, the closed-loop control system would become $\hat{D}(q)\left(\dot{\hat{s}}(t)+\Lambda_{1} \hat{s}(t)\right)=$ 0 . To guarantee the desired properties that $\hat{s}(t)$ is bounded and $\lim _{t \rightarrow \infty} \hat{s}(t)=0$ exponentially, the estimate $\hat{D}(q)$ of $D(q)$ needs to be nonsingular for all time $t$ during the adaptation process. We know that the matrix $\hat{D}(q)$ will remain positive definite and invertible if we insure that all parameters $d_{i j}$ remain within a sufficiently small range near the true values.

1With this motivation, we use parameter projection to restrict our estimates of the parameters to lie within bounds, and correspondingly, the adaptive law for $\theta_{D}(t)$ is redesigned as

$$
\dot{\theta}_{D}(t)=-\Gamma_{D} Y_{D}^{T}(q, \dot{\hat{s}}) \tilde{s}(t)+f_{D}(t)
$$

where $\Gamma=\operatorname{diag}\left\{\gamma_{D 1}, \gamma_{D 2}, \ldots, \gamma_{D n_{D}}\right\}$, with $\gamma_{D i}>0$ for $i=1,2, \ldots, n_{D}$, and $f_{D}(t)=$ $\left[f_{D 1}(t), \ldots, f_{D n_{D}}(t)\right]^{T}$ is the parameter projection vector whose components $f_{D i}(t)$ are designed as follows.

Denote $\theta_{D}^{*}=\left[\theta_{D 1}^{*}, \ldots, \theta_{D n_{D}}^{*}\right]^{T} \in R^{n_{D}}$, and let $\theta_{D i}^{*} \in\left[\theta_{D i}^{a}, \theta_{D i}^{b}\right]$, where $\theta_{D i}^{a}, \theta_{D i}^{b}$ are bounds of the unknown parameter $\theta_{D i}^{*}$ such that the following assumption holds:

Assumption 3.1. For any parameter $\theta_{D i} \in\left[\theta_{D i}^{a}, \theta_{D i}^{b}\right], i=1,2, \ldots, n_{D}$, the parameter vector $\theta_{D}=\left[\theta_{D 1}, \ldots, \theta_{D_{n_{D}}}\right]^{T}$ ensures that the estimate $D(q)=\hat{D}\left(q(t), \theta_{D}\right)$ is nonsingular for all $t \geq 0$.

Projection signals. The parameter bounds $\theta_{D i}^{a}, \theta_{D i}^{b}$ and partial knowledge of 
the parameters of $D(q)$ can be obtained for a specific manipulator system. We first denote a signal $g_{D}(t)$ using the knowledge of the parameter bounds $\theta_{D i}^{a}$ and $\theta_{D i}^{b}$ as

$$
g_{D}(t)=-\Gamma_{D} Y_{D}^{T}(q, \dot{\hat{s}}) \tilde{s}(t)=\left[g_{D 1}(t), g_{D 2}(t), \ldots, g_{D n_{D}}(t)\right]^{T}
$$

and then choose $\theta_{D i}(0) \in\left[\theta_{D i}^{a}, \theta_{D i}^{b}\right], i=1,2, \ldots, n_{D}$. Now we can set the projection signals $f_{D i}(t)$ as

$$
f_{D i}(t)= \begin{cases}0 & \text { if } \theta_{D i}(t) \in\left(\theta_{D i}^{a}, \theta_{D i}^{b}\right), \text { or } \\ & \text { if } \theta_{D i}(t)=\theta_{D i}^{a}, g_{D i}(t) \geq 0, o r \\ & \text { if } \theta_{D i}(t)=\theta_{D i}^{b}, g_{D i}(t) \leq 0 \\ -g_{D i}(t) & \text { if otherwise. }\end{cases}
$$

Choosing $f_{D i}(t)$ as above ensures that the estimate $\theta_{D i}(t) \in\left[\theta_{D i}^{a}, \theta_{D i}^{b}\right], i=$ $1,2, \ldots, n_{D}$, so that $\theta_{D}(t) \in L^{\infty}$. Furthermore, this choice of $f_{D i}(t)$ guarantees that

$$
\left(\theta_{D i}(t)-\theta_{D i}^{*}\right) f_{D i}(t) \leq 0,1 \leq j \leq n_{D}
$$

so that with $\tilde{\theta}_{D i}(t)=\theta_{D i}(t)-\theta_{D i}^{*}$ and $\tilde{\theta}_{D}(t)=\left[\tilde{\theta}_{D 1}(t), \ldots, \tilde{\theta}_{D n_{D}}(t)\right]^{T}=\theta_{D}(t)-\theta_{D}^{*}$, we can conclude that

$$
\tilde{\theta}_{D}^{T}(t) \Gamma_{D}^{-1} f_{D}(t)=\sum_{j=1}^{n_{\theta}} \tilde{\theta}_{D j}(t) \gamma_{D j}^{-1} f_{D j}(t) \leq 0
$$

With this parameter projection scheme, the following desired properties can be guaranteed.

Lemma 3.2. The adaptive parameter estimation scheme consisting of (3.20), (3.21) 
and (3.48) with (3.49)-(3.50) ensures that the prediction errors $\tilde{s}(t)=s(t)-\hat{s}(t)$, the parameter errors $\theta_{D}(t)-\theta_{D}^{*}, \theta_{D}(t)-\theta_{C}^{*}$ and $\theta(t)-\theta^{*}$ are bounded, $x \tilde{s}(t) \in L^{2}$, and $\hat{D}(q)=\hat{D}\left(q(t), \theta_{D}(t)\right)$ is nonsingular for all $t \geq 0$.

Proof: From the parameter projection property we have $\theta_{D i}(t) \in\left[\theta_{D i}^{a}, \theta_{D i}^{b}\right], i=$ $1,2, \ldots, n_{D}$, this property leads to a nonsingular $\hat{D}(q)$ as specified in Assumption 3.1 from the conditions on the intervals $\left[\theta_{D i}^{a}, \theta_{D i}^{b}\right]$, and from the positive definite function $V\left(\tilde{s}, \tilde{\theta}_{D}, \tilde{\theta}_{D}, \tilde{\theta}\right)$ in $(3.22)$, which has the property that its time-derivative as

$$
\dot{V}=-\tilde{s}^{T}(t) K_{0} \tilde{s}(t)+\tilde{\theta}_{D}^{T} \Gamma_{D}^{-1} f_{D}(t)
$$

With the property (3.52) of parameter projection, we have $\dot{V} \leq-\tilde{s}^{T}(t) K_{0} \tilde{s}(t)$, so that the desired properties of Lemma (3.1).

Notice that in the parametrization (3.11): $D(q)=\left\{d_{i j}(q)\right\}, d_{i j}(q)=\theta_{i j}^{* T} \phi_{i j}(q)$, the specific forms of the function $\phi_{i j}(q)$ are not unique, instead, they depend on a specific manipulator system model, and so do the parameter bounds $\theta_{D k}^{a}$ and $\theta_{D k}^{b}$ of the true parameter component $\theta_{D k}^{*}$ of $\theta_{D}^{*}$ (which is the bounds of the components of $\theta_{i j}^{*}$ ). Such functions and associated parameter bounds may not be unique for a parametrization scheme (3.11). An ideal design should choose $\theta_{i j}^{*}$ and $\phi_{i j}(q)$ with as lower dimensions as possible.

\subsection{Adaptive Controller}

With the parameter estimation scheme and parameter projection we proposed in section 3.2 , an adaptive control signal $u(t)$ can be chosen as

$$
u(t)=Y\left(q, q_{d}, \dot{q}, \dot{q}_{d}, \ddot{q}_{d}\right) \theta(t)-K_{0} \tilde{s}-\hat{D}(q) \Lambda_{1} \hat{s}(t)+\hat{C}(q, \dot{q}) \hat{s}(t)
$$


where $\hat{D}(q)$ is constructed as in $(3.14)$ and $\hat{C}(q, \dot{q}) \hat{s}(t)=Y_{C}(q, \dot{q}, \hat{s}) \theta_{C}$ as parametrized in $(3.16)$.

This choice of adaptive law guarantees the desired closed-loop properties for an adaptive control manipulator system, which are stated in the following theorem.

Theorem 3.1. The adaptive control law (3.54), updated by the adaptive parameter estimation scheme consisting of (3.48), (3.20) and (3.21), combined with the prediction algorithm (3.7) applied to the manipulator system (2.3), ensures that all signals in the closed-loop system are bounded and the tracking error $e(t)=q(t)-q_{d}(t)$ converges to zero as time goes to infinity.

Proof: By applying this control law, the prediction signal equation (3.7) becomes

$$
\hat{D}(q)\left(\dot{\hat{s}}+\Lambda_{1} \hat{s}(t)\right)=0 \text {. }
$$

With parameter projection, we could ensure that $\hat{D}(q)$ is positive definite and invertible for all $t \geq 0$, so that

$$
\dot{\hat{s}}(t)+\Lambda_{1} \hat{s}(t)=0
$$

from which we can conclude that $\hat{s}(t)$ is bounded and $\lim _{t \rightarrow \infty}=0$ exponentially, in other words, we obtain $\hat{s}(t) \in L^{2}$.

Consequently, the prediction signal $s(t)=\tilde{s}(t)+\hat{s}(t)$ is bounded, and $s(t) \in L^{2}$, since both $\tilde{s}(t)$ and $\hat{s}(t)$ have same properties. From the variable transformation $s=\dot{e}+\Lambda e$, we obtain that $e(t)$ is bounded and $e(t) \in L^{2}, \dot{e}(t)$ is bounded and $\dot{e}(t) \in L^{2}$, and in turn $u(t)$ in (3.54) is bounded. Finally, the properties that $e(t) \in L^{2}$ and $\dot{e}(t)$ is bounded lead to $\lim _{t \rightarrow \infty} e(t)=0$. So the theorem holds for all $t \geq 0$. 


\subsection{Simulation Study}

In this section, we present some simulation results to verify the desired performance of our prediction error based adaptive control scheme in single-model cases. In our simulation study, we consider a 2-link planar manipulator as the controlled plant for the robot system modeling, parametrization, and simulation.

\subsubsection{Simulation System}

The manipulator configuration may be described as: there are two revolute joints, one on the top of the other, with joint angles $q_{1}, q_{2}$, and two links with masses $m_{1}$, $m_{2}$, lengths $l_{1}, l_{2}$, distances $l_{c 1}, l_{c 2}$ from the joints to the mass centers, and rotational inertias $I_{1}, I_{2}$.

System equations. The dynamics equation of the rigid robot manipulator is given in equation (2.3). For a 2-link planar manipulator used in our simulation study, a more specific model can be obtained as follows. The inertia matrix $D(q)$ should be:

$$
D(q)=\left[\begin{array}{ll}
d_{11} & d_{12} \\
d_{21} & d_{22}
\end{array}\right]
$$

with

$$
\begin{gathered}
d_{11}=m_{1} l_{c 1}^{2}+m_{2}\left(l_{1}^{2}+l_{c 2}^{2}+2 l_{1} l_{c 2} \cos \left(q_{2}\right)\right)+I_{1}+I_{2} \\
d_{12}=d_{21}=m_{2}\left(l_{c 2}^{2}+l_{1} l_{c 2} \cos \left(q_{2}\right)\right)+I_{2} \\
d_{22}=m_{2} l_{c 2}^{2}+I_{2} .
\end{gathered}
$$


The matrix $C(q, \dot{q})$ representing Coriolis and centrifugal effects should be:

$$
C(q)=\left[\begin{array}{ll}
c_{11} & c_{12} \\
c_{21} & c_{22}
\end{array}\right]
$$

with

$$
\begin{gathered}
c_{11}=-m_{2} l_{1} l_{c 2} \sin \left(q_{2}\right) \dot{q}_{2} \\
c_{12}=-m_{2} l_{1} l_{c 2} \sin \left(q_{2}\right)\left(\dot{q}_{1}+\dot{q}_{2}\right) \\
c_{21}=m_{2} l_{1} l_{c 2} \sin \left(q_{2}\right) \dot{q}_{1} \\
c_{22}=0 .
\end{gathered}
$$

The gravity matrix $\phi(q)$ should be:

$$
\phi(q)=\left[\begin{array}{c}
\left(m_{1} l_{c 1}+m_{2} l_{1}\right) g \cos \left(q_{1}\right)+m_{2} l_{c 2} g \cos \left(q_{1}+q_{2}\right) \\
m_{2} l_{c 2} g \cos \left(q_{1}+q_{2}\right)
\end{array}\right] .
$$

Parametrization. After using variable transformation (3.1) and parameterizing the manipulator model into (3.4), we have the parameter vector $\theta^{*}$ as

$$
\begin{aligned}
\theta^{*} & =\left[\theta_{1}^{*}, \theta_{2}^{*}, \theta_{3}^{*}, \theta_{4}^{*}, \theta_{5}^{*}\right] \\
& =\left[m_{1} l_{c 1}^{2}+m_{2} l_{1}^{2}+m_{2} l_{c 2}^{2}+I_{1}+I_{2}, m_{2} l_{c 2}^{2}+I_{2}, m_{2} l_{1} l_{c 2},\left(m_{1} l_{c 1}+m_{2} l_{1}\right) g, m_{2} l_{c 2} g\right]^{T}
\end{aligned}
$$


and the regressor matrix $Y\left(q, q_{d}, \dot{q}, \dot{q}_{d}, \ddot{q}_{d}\right)$ as

$$
\begin{gathered}
Y\left(q, q_{d}, \dot{q}, \dot{q}_{d}, \ddot{q}_{d}\right)=Y_{0}(q, \dot{q}, v, \dot{v})=\left[\begin{array}{lll}
\dot{v}_{1} & \dot{v}_{2} & \\
& & \\
0 & \dot{v}_{1}+\dot{v}_{2} & \\
\cos \left(q_{2}\right)\left(2 \dot{v}_{1}+\dot{v}_{2}\right)-\sin \left(q_{2}\right) \dot{q}_{2} v_{1}-\sin \left(q_{2}\right)\left(\dot{q}_{1}+\dot{q}_{2}\right) v_{2} & \cos \left(q_{1}\right) & \cos \left(q_{1}+q_{2}\right) \\
\cos \left(q_{2}\right) \dot{v}_{1}+\sin \left(q_{2}\right) \dot{q}_{1} v_{1} & 0 & \cos \left(q_{1}+q_{2}\right)
\end{array}\right]
\end{gathered}
$$

where $v=\left[v_{1}, v_{2}\right]^{T}$.

We may also parametrized $C(q, \dot{q}) \hat{s}$ and $D(q) \dot{\hat{s}}$ as

$$
\begin{gathered}
C(q, \dot{q}) \hat{s}=Y_{C}(q, \dot{q}, \hat{s}) \theta_{C}^{*} \\
=\left[\begin{array}{c}
\left.-\sin \left(q_{2}\right) \dot{q}_{2} \hat{s}_{1}-\sin \left(q_{2}\right)\left(\dot{q}_{1}+\dot{q}_{2}\right) \hat{s}_{2}\right] \\
\sin \left(q_{2}\right) \dot{q}_{1} \hat{s}_{1}
\end{array}\right]\left(m_{2} l_{1} l_{c 2}\right) \\
D(q) \dot{\hat{s}}=Y_{D}(q, \dot{\hat{s}}) \theta_{D}^{*} \\
=\left[\begin{array}{cc}
\dot{\hat{s}}_{1} & \dot{\hat{s}}_{2} \\
0 & 2 \dot{\hat{s}}_{1} \cos \left(q_{2}\right)+\dot{\hat{s}}_{2} \cos \left(q_{2}\right) \\
\dot{\hat{s}}_{2} & \dot{\hat{s}}_{1} \cos \left(q_{2}\right)
\end{array}\right]\left[\begin{array}{c}
m_{1} l_{c 1}^{2}+m_{2} l_{1}^{2}+m_{2} l_{c 2}^{2}+I_{1}+I_{2} \\
m_{2} l_{c 2}^{2}+I_{2} \\
m_{2} l_{1} l_{c 2}
\end{array}\right] .
\end{gathered}
$$

Note that for a 2-link robot manipulator, parametrization of $D(q) \dot{\hat{s}}$ can be simplified into three parameters instead of five as above.

Control signal. The control input signal $u(t)$ we choose for this robot system is given in (3.54): $u(t)=Y\left(q, q_{d}, \dot{q}, \dot{q}_{d}, \ddot{q}_{d}\right) \theta(t)-K_{d} \tilde{s}(t)-\hat{D}(q) \Lambda_{1} \hat{s}(t)+\hat{C}(q, \dot{q}) \hat{s}(t)$. 
System parameters. The nominal values for the inertial parameters can be obtained as follows: $m_{1}=6.5225 \mathrm{~kg}, m_{2}=2.0458 \mathrm{~kg}, l_{1}=l_{2}=0.26 \mathrm{~m}, l_{c 1}=0.0983 \mathrm{~m}$, $l_{c 2}=0.0229 \mathrm{~m}, I_{1}=0.1213 \mathrm{kgm}^{2}$, and $I_{2}=0.0116 \mathrm{kgm}^{2}$. Hence we could get the true values of parameter vectors $\theta^{*}, \theta_{D}^{*}$ and $\theta_{C}^{*}$ :

$$
\begin{aligned}
& \theta^{*}=\left[\begin{array}{lllll}
0.3353 & 0.0127 & 0.0122 & 11.5078 & 0.4596
\end{array}\right]^{T} \\
& \theta_{D}^{*}=\left[\begin{array}{lll}
0.3353 & 0.0127 & 0.0122
\end{array}\right]^{T} \\
& \theta_{C}^{*}=0.0122 .
\end{aligned}
$$

\subsubsection{Simulation Results}

In the simulation study, we tested our prediction error based adaptive control scheme in two cases, where different desired trajectories were used to verify the tracking error convergence. The simulation results show that in both cases, our control objective could be achieved.

Simulation conditions. We assumed the robot manipulator is executing a trajectory-tracking task in our tests. The initial joint positions were set as $q_{1}(0)=$ $\pi / 6, q_{2}(0)=\pi / 3$ for both cases. Initial values of other parameters were chosen as $\dot{q}_{1}(0)=\pi / 4, \dot{q}_{2}(0)=\pi / 4, \hat{s}_{1}(0)=1, \hat{s}_{2}(0)=1, \theta_{1}(0)=0.3, \theta_{2}(0)=0.01, \theta_{3}(0)=0.01$, $\theta_{4}(0)=10, \theta_{5}(0)=1, \theta_{D 1}(0)=0.01, \theta_{D 2}(0)=0.01, \theta_{D 3}(0)=0.01, \theta_{C}(0)=0.01$. Choose design parameters as $K_{d}=I_{2}, \Lambda=I_{2}$, and $\Lambda_{1}=0.8 I_{2}$, and adaptive gains as $\Gamma_{D}=0.1 I_{3}, \Gamma_{C}=0.1 I_{1}, \Gamma=10 I_{5}$ for simulation.

Notice that for the purpose of parameter projection, we need to restrict $\hat{\theta}_{D 1}, \hat{\theta}_{D 2}$ and $\hat{\theta}_{D 3}$ to lie on chosen ranges so that $\hat{D}(q)$ remains invertible. Based on priori 
knowledge, we set the parameter bounds as

$$
\begin{gathered}
0.28<\hat{\theta}_{D 1}<0.55 \\
0.0125<\hat{\theta}_{D 2}<0.0410 \\
0.0085<\hat{\theta}_{D 3}<0.0125 .
\end{gathered}
$$

In Case I, the desired trajectory was chosen as $q_{1 d}=\pi, q_{2 d}=\pi / 2$, testing the system performance in set-point control. In Case II, the desired trajectories were chosen as $q_{1 d}(t)=\pi / 3+\sin (t), q_{2 d}(t)=\pi / 6+\cos (t)$ along with their first and second derivatives.

System responses. The simulation results of Case I are shown in Figures 3.1 3.3, and results of Case II are shown in Figures 3.4 - 3.6. According to the system responses, we found that in both cases, with the adaptive control laws and the prediction error based algorithm applied to the manipulator system, the tracking errors $e(t)=q(t)-q_{d}(t)$ converge to zero, and the parameter estimation errors $\theta(t)-\theta^{*}$, $\theta_{D}(t)-\theta_{D}^{*}, \theta_{C}(t)-\theta_{C}^{*}$ are all bounded. 


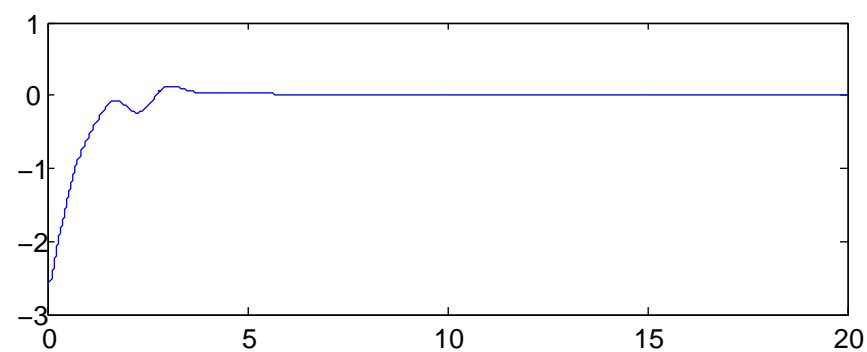

(a) Tracking error $\mathrm{e}_{1}(\mathrm{t})$ in $\mathrm{rad} / \mathrm{sec}$

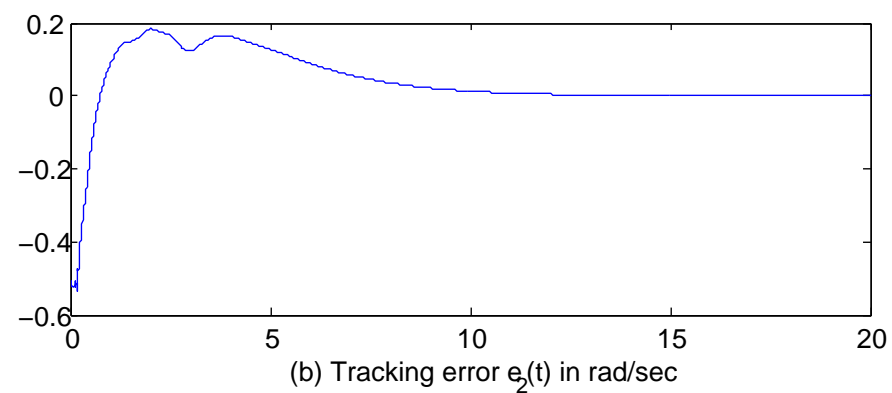

Figure 3.1: Tracking errors $e(t)=q(t)-q_{d}(t)$ for the single-model scheme (Case I).

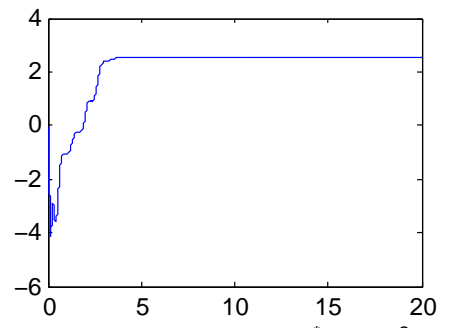

(a) Parameter error $\theta_{1}-\theta_{1}^{*}$ in $\mathrm{kgm}^{2} / \mathrm{sec}$

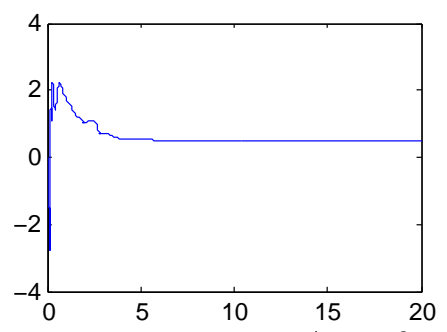

(b) Parameter error $\theta_{2}-\theta_{2}^{*}$ in $\mathrm{kgm}^{2} / \mathrm{sec}$

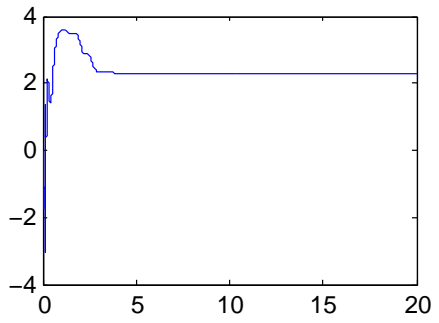

(c) Parameter error $\theta_{3}-\theta_{3}^{*}$ in $\mathrm{kgm}^{2} / \mathrm{sec}$

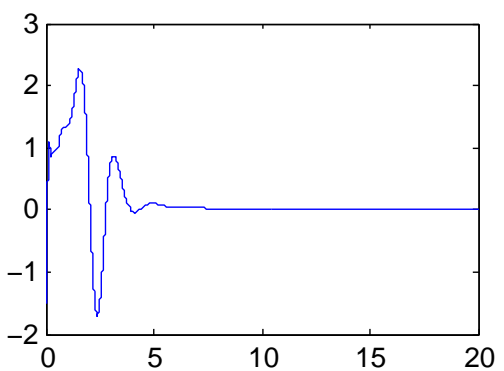

(d) Parameter error $\theta_{4}-\theta_{4}^{*}$ in $\mathrm{kgm}^{2} / \mathrm{sec}$

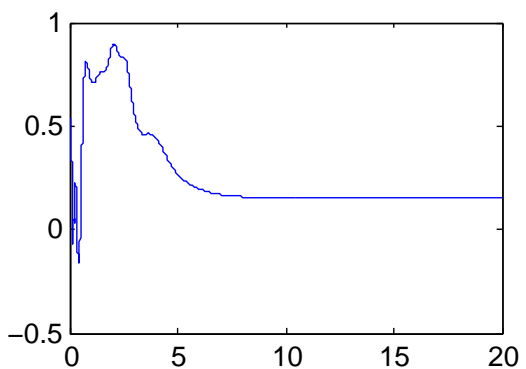

(e) Parameter error $\theta_{5}-\theta_{5}^{*}$ in $\mathrm{kgm}^{2} / \mathrm{sec}$

Figure 3.2: Parameter errors of $\theta(t)$ for the single-model scheme (Case I). 


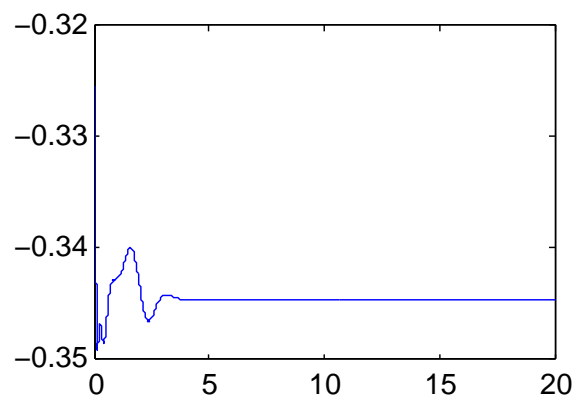

(a) Parameter error $\theta_{\mathrm{D} 1}-\theta_{\mathrm{D} 1}^{*}$ in $\mathrm{kgm}^{2} / \mathrm{sec}$

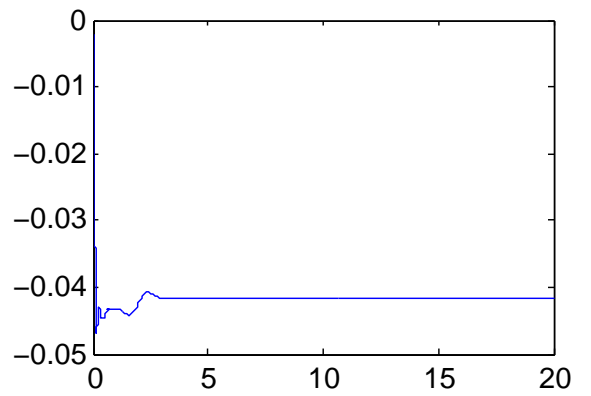

(c) Parameter error $\theta_{\mathrm{D} 3}-\theta_{\mathrm{D} 3}^{*}$ in $\mathrm{kgm}^{2} / \mathrm{sec}$

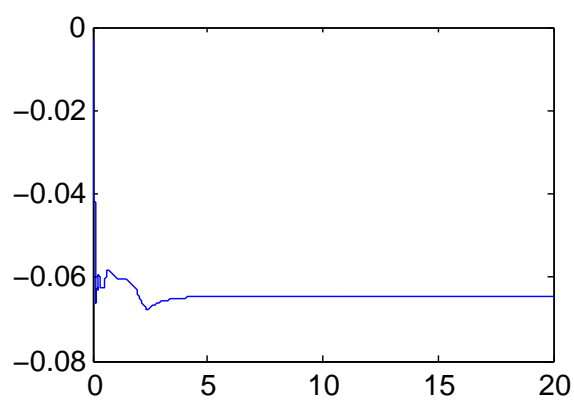

(b) Parameter error $\theta_{\mathrm{D} 2}-\theta_{\mathrm{D} 2}^{*}$ in $\mathrm{kgm}^{2} / \mathrm{sec}$

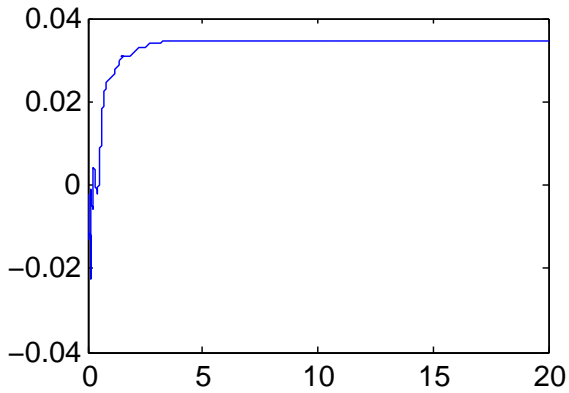

(d) Parameter error $\theta_{\mathrm{C}}-\theta_{\mathrm{C}}^{*}$ in $\mathrm{kgm}^{2} / \mathrm{sec}$

Figure 3.3: Parameter errors of $\theta_{D}(t)$ and $\theta_{C}(t)$ for the single-model scheme (Case I).

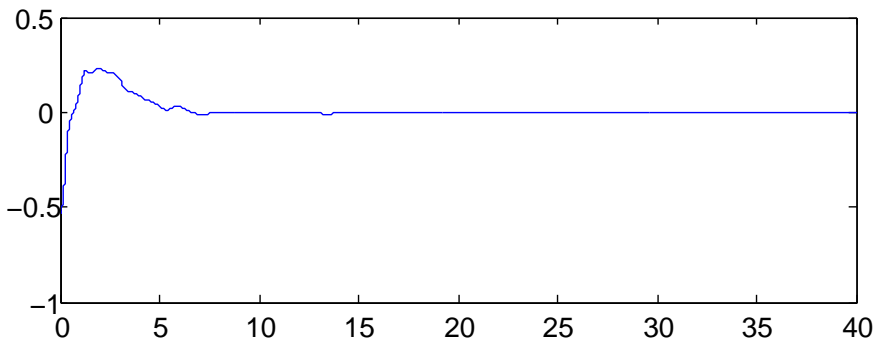

(a) Tracking error $\mathrm{e}_{4}(\mathrm{t})$ in $\mathrm{rad} / \mathrm{sec}$

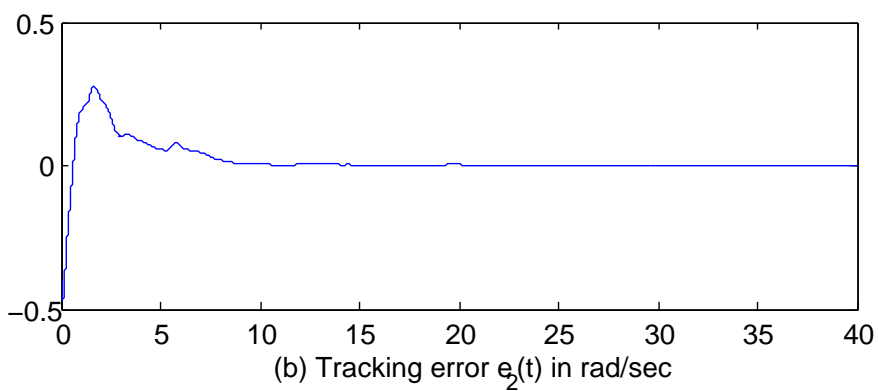

Figure 3.4: Tracking errors $e(t)=q(t)-q_{d}(t)$ for the single-model scheme (Case II). 


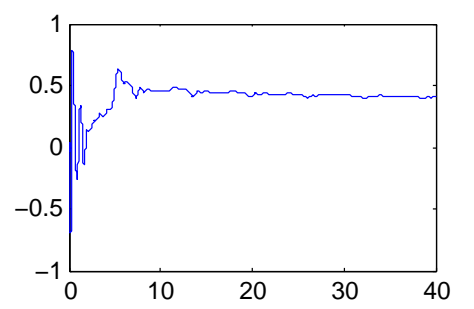

(a) Parameter error $\theta_{1}-\theta_{1}^{*}$ in $\mathrm{kgm}^{2} / \mathrm{sec}$

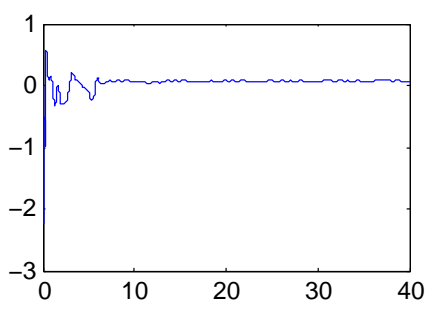

(b) Parameter error $\theta_{2}-\theta_{2}^{*}$ in $\mathrm{kgm}^{2} / \mathrm{sec}$

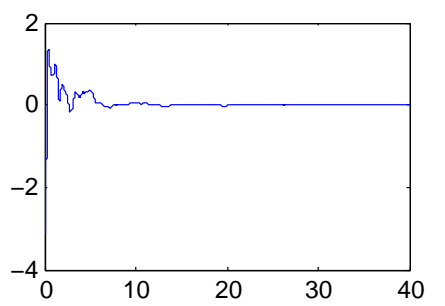

(c) Parameter error $\theta_{3}-\theta_{3}^{*}$ in $\mathrm{kgm}^{2} / \mathrm{sec}$

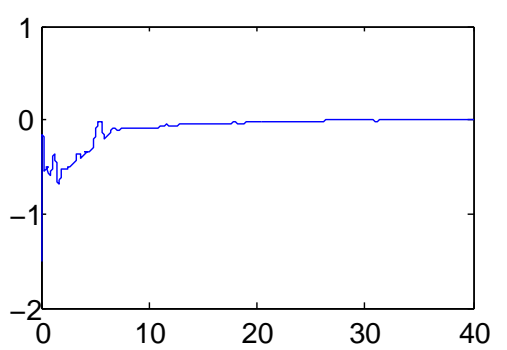

(d) Parameter error $\theta_{4}-\theta_{4}^{*}$ in $\mathrm{kgm}^{2} / \mathrm{sec}$

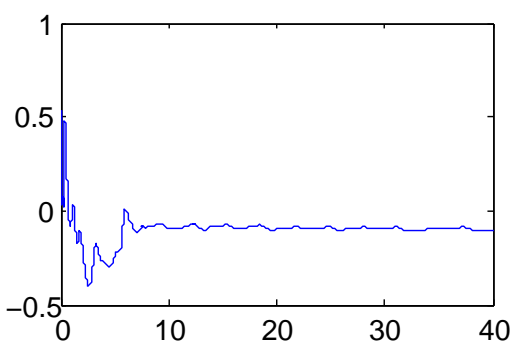

(e) Parameter error $\theta_{5}-\theta_{5}^{*}$ in $\mathrm{kgm}^{2} / \mathrm{sec}$

Figure 3.5: Parameter errors of $\theta(t)$ for the single-model scheme (Case II).

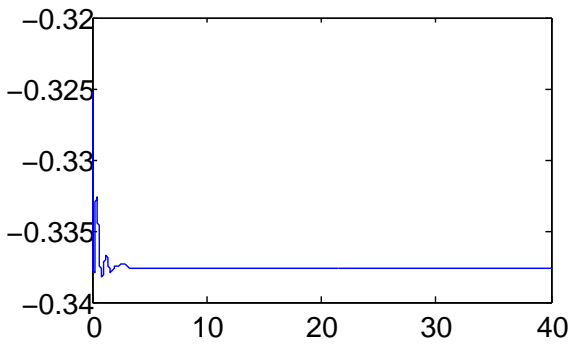

(a) Parameter error $\theta_{D 1}-\theta_{D 1}^{*}$ in $\mathrm{kgm}^{2} / \mathrm{sec}$

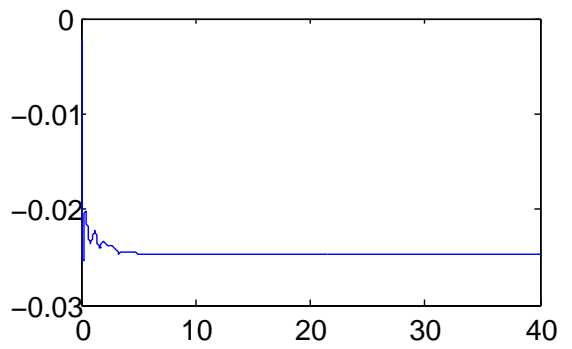

(c) Parameter error $\theta_{\mathrm{D} 3}-\theta_{\mathrm{D} 3}^{*}$ in $\mathrm{kgm}^{2} / \mathrm{sec}$

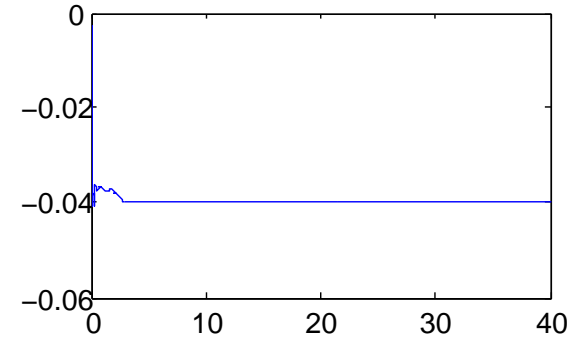

(b) Parameter error $\theta_{\mathrm{D} 2}-\theta_{\mathrm{D} 2}^{*}$ in $\mathrm{kgm}^{2} / \mathrm{sec}$

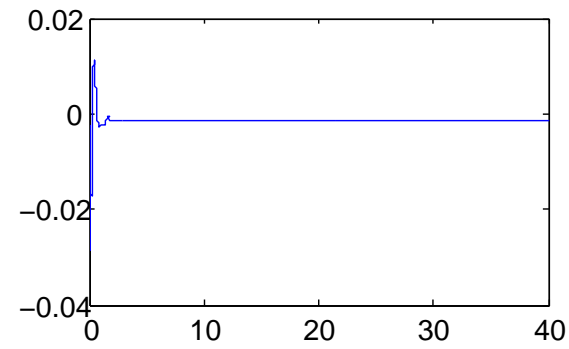

(d) Parameter error $\theta_{C}-\theta_{C}^{*}$ in $\mathrm{kgm}^{2} / \mathrm{sec}$

Figure 3.6: Parameter errors of $\theta_{D}(t)$ and $\theta_{C}(t)$ for the single-model scheme (Case II). 


\subsection{Simulation Discussion and Summary}

From the simulation results shown in Figure 3.1 - 3.6, we found that the proposed adaptive control scheme is effective in solving the control problem of robotic manipulators executing trajectory tracking tasks. In Case I, the tracking errors and parameter errors converge after 6 seconds. And in Case II, it takes around 10 seconds. We also notice that there are undesirable overshoots in the system responses. For tracking errors, overshoots are around $0.2 \mathrm{rad} / \mathrm{sec}$ in Case I, and $0.3 \mathrm{rad} / \mathrm{sec}$ in Case II. It is our goal to improve the system performance by expanding this prediction error based adaptive control scheme to multiple-model control scheme.

The dynamic prediction error based adaptive control scheme we designed in this chapter contains the basic structure for development to multiple-model control scheme, which has the potential for system performance improvement. As a result, the method and steps of parameter estimation, compact parametrization, and adaptive controller design will be followed in our multiple-model design in next chapter. The parameter projection scheme designed in this chapter will also play an important role in stability analysis for multiple-model control scheme. 


\section{Chapter 4}

\section{A Multiple-Model Adaptive Control Scheme}

In this chapter, our objective is to devise an algorithm for the trajectory tracking of robotic manipulators, which deal with uncertain environmental conditions. What different from the content of last chapter is an additional constraint that the transient behavior of the tracking error is as desired as we want. In order to achieve improvement in overall performance, we use the adaptive control scheme proposed in last chapter as a baseline to develop a multiple-model control scheme. Simulation study is also presented in this chapter to verify the improvement of transient performance.

\subsection{Problem Statement}

From the content of last chapter, we know that the main technical issue of designing an adaptive control scheme is that due to the uncertainty of $\theta^{*}$, we cannot decide which is the most suitable choice of the initial parameter estimate $\theta(0)$ and the adaptation gain matrix $\Gamma$. Although the desired control system stability (signal boundedness) and asymptotic tracking $\lim _{t \rightarrow \infty} e(t)=0$ of the adaptive control scheme 
can always be guaranteed whatever $\theta^{*}$ and $\Gamma$ we choose, different choices would lead to different system transient responses.

Our goal is to develop a multiple-model based adaptive control scheme for the robot manipulator system (2.3), which can incorporate different initial conditions $\theta(0)$ and different adaptation gains $\Gamma$ to select a most suitable control signal among the chosen collections. With a particular $\theta(0)$ and a particular $\Gamma$, improved system performance would be achieved, as compared with a single-model adaptive control scheme.

\subsection{Multiple-Model Adaptive Design}

Adaptive prediction signals. Based on the plant signal identity we proposed in (3.4), multiple adaptive prediction signals can be first designed as

$$
\hat{D}_{(i)}(q) \dot{\hat{s}}_{(i)}+\hat{C}_{(i)}(q, \dot{q}) \hat{s}_{(i)}=u-Y\left(q, q_{d}, \dot{q}, \dot{q}_{d}, \ddot{q}_{d}\right) \theta_{(i)}+K_{0(i)} \tilde{s}_{(i)},
$$

with multiple adaptive estimates $\hat{D}_{(i)}(q)$ and $\hat{C}_{(i)}(q, \dot{q})$ of $D(q)$ and $C(q, \dot{q})$, multiple estimates $\theta_{(i)}(t)$ of $\theta^{*}$, and with multiple $n \times n$ gain matrices $K_{0(i)}=K_{0(i)}^{T}>0$, where $\tilde{s}_{(i)}(t)=s(t)-\hat{s}_{(i)}(t)$ are the multiple prediction error signals, $i=1,2, \ldots, N$.

Notice that the initial values of the estimates $\hat{D}_{(i)}(q), \hat{C}_{(i)}(q, \dot{q})$ and $\theta_{(i)}(t)$ at $t=0$ should be chosen from different representative locations in the parameter space, and a desired choice of the number $N$ of the multiple controllers can be determined according to a specific application system.

According to the prediction signals (4.1) and (3.4), we can obtain the multiple 
prediction error equations

$D(q) \dot{\tilde{s}}_{(i)}+C(q, \dot{q}) \tilde{s}_{(i)}=\tilde{D}_{(i)}(q) \dot{\hat{s}}_{(i)}+\tilde{C}_{(i)}(q, \dot{q}) \hat{s}_{(i)}+Y\left(q, q_{d}, \dot{q}, \dot{q}_{d}, \ddot{q}_{d}\right)\left(\theta_{(i)}-\theta^{*}\right)-K_{0(i)} \tilde{s}_{(i)}$

where

$$
\tilde{D}_{(i)}(q)=\hat{D}_{(i)}(q)-D(q), \tilde{C}_{(i)}(q, \dot{q})=\hat{C}_{(i)}(q, \dot{q})-C(q, \dot{q})
$$

Parametrization of $\hat{C}_{(i)}(q, \dot{q}) \hat{s}_{(i)}$ and $\hat{D}_{(i)}(q) \dot{\hat{s}}_{(i)}$. Similar to the parametrization scheme presented in Section 3.2.1, which is (3.10): $C(q, \dot{q}) \hat{s}=Y_{C}(q, \dot{q}, \tilde{s}) \theta_{C}^{*}$ and (3.13): $D(q) \dot{\hat{s}}=Y_{D}(q, \dot{\hat{s}}) \theta_{D}^{*}$, we can express the parametrization in multiple-model scheme as

$$
\begin{aligned}
C(q, \dot{q}) \hat{s}_{(i)} & =Y_{C}\left(q, \dot{q}, \hat{s}_{(i)}\right) \theta_{C}^{*} \\
\hat{C}_{(i)}(q, \dot{q}) \hat{s}_{(i)} & =Y_{C}\left(q, \dot{q}, \hat{s}_{(i)}\right) \theta_{C(i)} \\
D(q) \dot{\hat{s}}_{(i)} & =Y_{D}\left(q, \dot{\hat{s}}_{(i)}\right) \theta_{D}^{*} \\
\hat{D}_{(i)}(q) \dot{\hat{s}}_{(i)} & =Y_{D}\left(q, \dot{\hat{s}}_{(i)}\right) \theta_{D(i)}
\end{aligned}
$$

where $\theta_{D(i)}$ and $\theta_{C(i)}$ are multiple estimates of $\theta_{D}^{*}$ and $\theta_{C}^{*}$. With these new expressions, the error equation (4.2) becomes

$$
\begin{aligned}
& D(q) \dot{\tilde{s}}_{(i)}+C(q, \dot{q}) \tilde{s}_{(i)} \\
= & Y_{D}\left(q, \dot{\hat{s}}_{(i)}\right)\left(\theta_{D(i)}-\theta_{D}^{*}\right)+Y_{C}\left(q, \dot{q}, \hat{s}_{(i)}\right)\left(\theta_{C(i)}-\theta_{C}^{*}\right) \\
& +Y\left(q, q_{d}, \dot{q}, \dot{q}_{d}, \ddot{q}_{d}\right)\left(\theta_{(i)}-\theta^{*}\right)-K_{0(i)} \tilde{s}_{(i)} .
\end{aligned}
$$

Adaptive laws. According to the parameter estimation and parameter projection scheme in last chapter, we choose the adaptive parameter update laws for $\theta_{D(i)}(t)$, 
$\theta_{C(i)}(t)$ and $\theta_{(i)}(t), i=1,2, \ldots, N$ as

$$
\begin{gathered}
\dot{\theta}_{D(i)}(t)=-\Gamma_{D(i)} Y_{D}^{T}\left(q, \dot{\hat{s}}_{(i)}\right) \tilde{s}_{(i)}(t)+f_{D(i)}(t) \\
\dot{\theta}_{C(i)}(t)=-\Gamma_{C(i)} Y_{C}^{T}\left(q, \dot{q}, \hat{s}_{(i)}\right) \tilde{s}_{(i)}(t) \\
\dot{\theta}_{(i)}(t)=-\Gamma_{(i)} Y^{T}\left(q, q_{d}, \dot{q}, \dot{q}_{d}, \ddot{q}_{d}\right) \tilde{s}_{(i)}(t),
\end{gathered}
$$

where $\Gamma_{D(i)}=\operatorname{diag}\left\{\gamma_{D(i) 1}, \gamma_{D(i) 2}, \ldots, \gamma_{D(i) n_{D}}\right\}$, with $\gamma_{D(i) j}>0$ for $j=1,2, \ldots, n_{D}, \Gamma_{C(i)} \in$ $R^{n_{C} \times n_{C}}$ and $\Gamma_{(i)} \in R^{r \times r}$ are such that $\Gamma_{C(i)}=\Gamma_{C(i)}^{T}>0$ and $\Gamma_{(i)}=\Gamma_{(i)}^{T}>0$, and $f_{D(i)}(t)=\left[f_{D(i) 1}(t), \ldots, f_{D(i) n_{D}}(t)\right]^{T}$ is the parameter projection vector whose components are defined in a way similar to that given in (3.50) to ensure that $\hat{D}_{(i)}(q)=\hat{D}_{(i)}\left(q(t), \theta_{D(i)}\right)$ is nonsingular for all $t$. Also, similar to the property we described in (3.52), for multiple-model scheme, we have

$$
\tilde{\theta}_{D(i)}^{T}(t) \Gamma_{D(i)}^{-1} f_{D(i)}(t)=\sum_{j=1}^{n_{\theta}} \tilde{\theta}_{D(i) j}(t) \gamma_{D(i) j}^{-1} f_{D(i) j}(t) \leq 0
$$

for the components $\tilde{\theta}_{D(i) j}(t)$ of $\tilde{\theta}_{D(i)}^{T}(t)$.

The initial parameters for multiple-model adaptive laws $\theta_{D(i)}(0), \theta_{C(i)}(0)$ and $\theta_{(i)}(0), i=1,2, \ldots, N$, are chosen at different representative locations in the parameter space where their true values $\theta_{D}^{*}, \theta_{D}^{*}$ and $\theta^{*}$ belong to. As a result, the minimum norm of the initial parameter errors $\theta_{D(i)}(0)-\theta_{D}^{*}, \theta_{C(i)}(0)-\theta_{C}^{*}$ and $\theta_{(i)}(0)-\theta^{*}$, $i=1,2, \ldots, N$, can be made as small as possible for uncertain parameters $\theta_{D}^{*}, \theta_{C}^{*}$, and $\theta^{*}$. To understand this claim, we consider the multiple sets of initial parameters $\left\{\theta_{D(i)}(0), \theta_{C(i)}(0), \theta_{(i)}(0)\right\}$, one set of them is the same with the one used for single-model adaptive controller, so that this set of initial parameters is as close to $\left(\theta_{D}^{*}, \theta_{C}^{*}, \theta^{*}\right)$ as that for a single-model scheme. Hence, with more sets of initial parameters being considered, some of $\left\{\theta_{D(i)}(0), \theta_{C(i)}(0), \theta_{(i)}(0)\right\}$ may be made closer to 
$\left(\theta_{D}^{*}, \theta_{C}^{*}, \theta^{*}\right)$, and a control switching mechanism may be eligible to choose the control signal with a closer set of initial parameters.

Adaptive control signals. The multiple adaptive control signals $u_{(i)}(t)$ is defined as

$$
u_{(i)}(t)=Y\left(q, q_{d}, \dot{q}, \dot{q}_{d}, \ddot{q}_{d}\right) \theta_{(i)}(t)-K_{0(i)} \tilde{s}_{(i)}(t)-\hat{D}_{(i)}(q) \Lambda_{1(i)} \hat{s}_{(i)}(t)+\hat{C}(q, \dot{q}) \hat{s}_{(i)}(t),
$$

where $\Lambda_{1(i)} \in R^{n \times n}$ is a constant matrix such that $-\Lambda_{1(i)}$ is stable, which requires the eigenvalues of this matrix all have negative real-part. $\hat{D}_{(i)}(q)$ and $\hat{C}_{(i)}(q, \dot{q}) \hat{s}_{(i)}(t)$ are obtained from

$$
\hat{D}_{(i)}(q)=\hat{D}_{(i)}\left(q, \theta_{D(i)}\right)=\left\{\hat{d}_{j k(i)}\left(q, \theta_{j k(i)}\right)\right\}
$$

where $\hat{d}_{j k(i)}\left(q, \theta_{j k(i)}\right)=\theta_{j k(i)}^{T} \phi_{j k}(q)$, and

$$
\hat{C}_{(i)}(q, \dot{q}) \hat{s}_{(i)}=Y_{C}\left(q, \dot{q}, \hat{s}_{(i)}\right) \theta_{C(i)}, i=1,2, \ldots, N
$$

Performance index. For the $i$ th prediction error $\tilde{s}_{(i)}(t)=s(t)-\hat{s}_{(i)}(t)$, we introduce the performance index $J_{(i)}(t)$ as follow:

$$
J_{(i)}(t)=\int_{0}^{t} e^{-\lambda(t-\tau)} \tilde{s}_{(i)}^{T}(\tau) \tilde{s}_{(i)}(\tau) d \tau, i=1,2, \ldots, N,
$$

for some constant $\lambda \geq 0$. We then set the control signal $u(t)$ at every instant as

$$
u(t)=u_{(i)}(t), i=\arg \min _{k=1,2, \ldots, N} J_{(k)}(t)
$$

That is, $u(t)$ is set to be $u_{(i)}(t)$ with $J_{(i)}(t)$ being the minimum of $J_{(k)}(t)$ for all $k=1,2, \ldots, N$. 


\subsection{Adaptive Control System Analysis}

In this section, we analyze the closed-loop system stability and output tracking properties of the above multiple-model adaptive control scheme applied to the manipulator system (2.3) with parameter uncertainties. We first show that the multiple parameter estimation scheme has the following desired properties.

Lemma 4.1. For each $i=1,2, \ldots, N$, the adaptive parameter estimator consisting of (4.9) with a parameter projection signal $f_{D(i)}(t)$, (4.10) and (4.11) ensures that $\tilde{s}_{(i)}(t)=s(t)-\hat{s}_{(i)}(t), \theta_{D(i)}(t)-\theta_{D}^{*}, \theta_{C(i)}(t)-\theta_{C}^{*}$ and $\theta_{(i)}(t)-\theta^{*}$ are bounded, $\tilde{s}_{(i)}(t) \in$ $L^{2}$, and $\hat{D}_{(i)(q)}=\hat{D}\left(q(t), \theta_{D(i)}(t)\right)$ is nonsingular for all $t \geq 0$.

Proof: For $i=1,2, \ldots, N$, from the parameter projection property: $\theta_{D(i) j}(t) \in$ $\left[\theta_{D j}^{a}, \theta_{D j}^{b}\right], j=1,2, \ldots, n_{D}$, we have a nonsingular $\hat{D}_{(i)}(q)$ from the conditions on the intervals $\left[\theta_{D j}^{a}, \theta_{D j}^{b}\right]$ as specified in Assumption 3.1. For the positive definite function

$V_{(i)}\left(\tilde{s}_{(i)}, \tilde{\theta}_{D(i)}, \tilde{\theta}_{C(i)}, \tilde{\theta}_{(i)}\right)=\frac{1}{2}\left(\tilde{s}_{(i)}^{T} D \tilde{s}_{(i)}+\tilde{\theta}_{D(i)}^{T} \Gamma_{D(i)}^{-1} \tilde{\theta}_{D(i)}+\tilde{\theta}_{C(i)}^{T} \Gamma_{C(i)}^{-1} \tilde{\theta}_{C(i)}+\tilde{\theta}_{(i)}^{T} \Gamma_{(i)}^{-1} \tilde{\theta}_{(i)}\right)$,

we derive its time-derivative as

$$
\dot{V}_{(i)}=-\tilde{s}_{(i)}^{T}(t) K_{0(i)} \tilde{s}_{(i)}(t)+\tilde{\theta}_{D(i)}^{T}(t) \Gamma_{D(i)}^{-1} f_{D(i)}(t)
$$

With the property (4.12) of parameter projection, we can obtain the desired property: $\dot{V}_{(i)} \leq-\tilde{s}_{(i)}^{T}(t) K_{0(i)} \tilde{s}_{(i)}(t) \leq 0$. From this result, it follows that $\tilde{s}_{(i)}(t)=s(t)-\hat{s}_{(i)}(t)$ $\theta_{D(i)}(t)-\theta_{D}^{*}, \theta_{C(i)}(t)-\theta_{C}^{*}$ and $\theta_{(i)}(t)-\theta^{*}$ are bounded, and that $\tilde{s}_{(i)} \in L^{2}$. These desired properties hold for all adaptive predictors (4.1) with parameter estimators (4.9), (4.10) and (4.11), for $i=1,2, \ldots, N$. 
Thus we have shown that each parameter estimator has the desired properties: $\theta_{D(i)}(t), \theta_{C(i)}(t)$ and $\theta_{(i)}(t)$ are bounded, $\hat{D}_{(i)}(q)=\hat{D}\left(q(t), \theta_{D(i)}(t)\right)$ is nonsingular for all $t \geq 0$, and $\tilde{s}_{(i)}(t) \in L^{2}$, for $i=1,2, \ldots, N$. Such multiple estimators generates parameters for implementing the multiple control signals $u_{(i)}(t)$ in (4.13). If $u(t)=$ $u_{(i)}(t), t \geq 0$, for any chosen $i$, the closed-loop system is stable and $\lim _{t \rightarrow \infty} e(t)=0$, as shown in Chapter 3.

Now we have the proof of stability for adaptive controllers without control switching, then a theorem should be stated to analyze the stability and tracking properties of the closed-loop system with multiple-model and switching control scheme.

Theorem 4.1. The multiple-model adaptive control scheme defined by the system equation (2.3), prediction error equations (4.8), control signals (4.13) updated by the adaptive laws (4.9)-(4.11), and the performance index (4.16) ensures that all signals in the closed-loop system are bounded, and the tracking errors $e(t)=q(t)-q_{d}(t)$ converge to zero as $t \rightarrow \infty$.

Proof: The proof of stability has similar procedures with proof in the single-model case, with the additional requirement that with the control switching mechanism driven by the performance index $J_{i}(t)$, all signals would remain bounded. Since Lemma 4.1 holds independently for every parameter estimator, which is used to generate the controller at any instant, the closed-loop stability can be guaranteed for every control signal $u_{i}(t)$, where $i=1,2, \ldots, N$. For the multiple-model scheme, the control switching mechanism is designed to improve the transient performance, without destabilizing the system, so that the parameter variations caused by control switching need to be small enough in an average sense. To achieve this goal, we use a waiting time $T_{\min }$ between successive control signal switches to prevent fast changing, which may cause system chattering. Also, we stop the control switching when $J_{i} \leq \epsilon_{0}$ for all $i=1,2, \ldots, N$ for some pre-chosen, arbitrary and small $\epsilon_{0}>0$. Thus, 
with the convergence of $J_{i}(t)$ to zero, there is a finite time $T^{*}$ such that the control switching stops for $t>T^{*}$, so the average switching frequency would be small, and the parameter variations caused by the control switching would be decreased as much as possible.

Moreover, when a control switch happens, a new control signal $u_{(j)}(t)$ is selected corresponding to the minimal cost function $J_{j}(t)$. That is, if $J_{j}\left(t_{1}\right)<J_{k}\left(t_{1}\right)$, the control switching mechanism switches from $u_{(k)}$ to $u_{(j)}$ at a time instant $t_{1}$, that is, the control signal change from

$$
\begin{aligned}
u(t) & =u_{(k)}(t) \\
& =Y\left(q, q_{d}, \dot{q}, \dot{q}_{d}, \ddot{q}_{d}\right) \theta_{(k)}(t)-K_{0} \tilde{s}_{(k)}(t)-\hat{D}_{(k)}(q) \Lambda_{1} \hat{s}_{(k)}(t)+\hat{C}_{(k)}(q, \dot{q}) \hat{s}_{(k)}(t)
\end{aligned}
$$

to

$$
\begin{aligned}
u(t) & =u_{(j)}(t) \\
& =Y\left(q, q_{d}, \dot{q}, \dot{q}_{d}, \ddot{q}_{d}\right) \theta_{(j)}(t)-K_{0} \tilde{s}_{(j)}(t)-\hat{D}_{(j)}(q) \Lambda_{1} \hat{s}_{(j)}(t)+\hat{C}_{(j)}(q, \dot{q}) \hat{s}_{(j)}(t),
\end{aligned}
$$

so that the change of the control signal caused by the control switching is

$$
\begin{aligned}
\Delta u(t)= & u_{(j)}(t)-u_{(k)}(t) \\
= & Y\left(\theta_{(j)}(t)-\theta_{(k)}(t)\right)-K_{0}\left(\tilde{s}_{(j)}(t)-\tilde{s}_{(k)}(t)\right)-\Lambda_{1}\left(\hat{D}_{(j)} \hat{s}_{(j)}(t)-\hat{D}_{(k)} \hat{s}_{(k)}(t)\right) \\
& +\left(\hat{C}_{(j)} \hat{s}_{(j)}-\hat{C}_{(k)} \hat{s}_{(k)}\right) \\
= & Y\left(\theta_{(j)}(t)-\theta_{(k)}(t)\right)-K_{0}\left(\tilde{s}_{(j)}(t)-\tilde{s}_{(k)}(t)\right)-\Lambda_{1}\left(Y_{D(j)} \theta_{D(j)}(t)-Y_{D(k)} \theta_{D(k)}(t)\right) \\
& +\left(Y_{C(j)} \theta_{C(j)}(t)-Y_{C(k)} \theta_{C(k)}(t)\right) .
\end{aligned}
$$


From Lemma 4.1, we have that $\tilde{s}_{(i)}, \theta_{(i)}(t)-\theta^{*}, \theta_{D(i)}(t)-\theta_{D}^{*}$ and $\theta_{C(i)}(t)-\theta_{C}^{*}$ are all bounded, and $\tilde{s}_{(i)}(t) \in L^{2}$ for all $t \geq 0$, so the variations of the control signal during control switching are small. As a result, the desired signal boundedness property in Lemma 4.1 will also holds for multiple-model scheme under the control switching. In other words, the $L^{2}$ properties of the adaptive laws given in Lemma 4.1 is reserved under the control switching mechanism.

Since the parameter projection works under multiple-model and switching scheme, $\hat{D}_{(i)}(q)=\hat{D}\left(q(t), \theta_{D(i)}(t)\right)$ remains nonsingular during the switching process. With $\tilde{s}_{(i)}(t) \in L^{2}$ always holds, we conclude that the control input signal $u(t)=u_{(i)}(t)$, generated from the parameter estimators are also bounded, is also bounded under switching, which results in the closed-loop stability, together with the tracking error $\lim _{t \rightarrow \infty} e(t)=0$.

\subsection{Comparison Study}

From what we have discussed in this chapter, the basic idea of multiple-model adaptive control is that when dealing with parameter uncertainties, the use of control signal switching among multiple controllers has the potential to be more effective than single-model adaptive control. This is the reason why multiple-model is considered as a method to improve system stability and tracking performance.

In the single-model control scheme, a prediction error signal $\tilde{s}(t)$ generated in (3.8) can be used to characterize certain system error in the presence of the uncertainty of the parameters $\theta_{D}^{*}, \theta_{C}^{*}$ and $\theta^{*}$. If it happens to be $\theta_{D}(t)=\theta_{D}^{*}, \theta_{C}(t)=\theta_{C}^{*}$ and $\theta(t)=\theta^{*}$, then it follow from (3.8) that

$$
D(q) \dot{\tilde{s}}+C(q, \dot{q}) \tilde{s}=-K_{0} \tilde{s}
$$


which leads to $\lim _{t \rightarrow \infty} \tilde{s}(t)=0$ exponentially. When $\theta_{D}(t) \neq \theta_{D}^{*}, \theta_{C}(t) \neq \theta_{C}^{*}$ and $\theta(t) \neq \theta^{*}$, the prediction error signal $\tilde{s}(t)$ also satisfies equation $(3.8): D(q) \dot{\tilde{s}}(t)+$ $C(q, \dot{q}) \tilde{s}=\tilde{D}(q) \dot{\hat{s}}+\tilde{C}(q, \dot{q}) \hat{s}+Y\left(q, q_{d}, \dot{q}, \dot{q}_{d}, \ddot{q}\right)\left(\theta-\theta^{*}\right)-K_{0} \tilde{s}$. What more important is that $\tilde{s}(t)$ depends on the parameter errors (also called parameter uncertainties) $\theta_{D}(t)-$ $\theta_{D}^{*}, \theta_{C}(t)-\theta_{C}^{*}$ and $\theta(t)-\theta^{*}$. In other words, the effect of the parameter uncertainties is reflected by the prediction error signal $\tilde{s}(t)$. Generally, smaller parameter errors $\theta_{D}(t)-\theta_{D}^{*}, \theta_{C}(t)-\theta_{C}^{*}$ and $\theta(t)-\theta^{*}$ lead to a smaller prediction error signal $\tilde{s}(t)$. However, "generally true" represents that this scenario happens almost usually but not always necessarily. The adaptive laws for updating the parameters $\theta_{D}(t), \theta_{C}(t)$ and $\theta(t)$ are chosen to ensure the properties stated in Lemma 3.1, and the eventual convergence: $\lim _{t \rightarrow \infty} \tilde{s}(t)=0$. In this sense, the smaller $\tilde{s}(t)$ is, the smaller parameter uncertainty would be. Since the prediction error $\tilde{s}(t)$ is the only way to quantitatively characterize or measure the parameter uncertainties by a known signal, we have to compromise on the fact that the improvement of transient performance may not be always necessarily true.

The value of the prediction error $\tilde{s}(t)$ depends on the initial parameter values $\theta_{D}(0), \theta_{C}(0)$ and $\theta(0)$ of the adaptive law for $\theta_{D}(t), \theta_{C}(t)$ and $\theta(t)$. From what we have discussed above, it is generally that the closer the initial values $\theta_{D}(0), \theta_{C}(0)$ and $\theta(0)$ are to the true values $\theta_{D}^{*}, \theta_{C}^{*}$ and $\theta^{*}$, the smaller the prediction error $\tilde{s}(t)$ would be. Hence, multiple-model adaptive control generate multiple prediction errors $\tilde{s}_{(i)}(t), i=$ $1,2, \ldots, N$, to provide more measurements of the uncertainties of $\theta_{D}^{*}, \theta_{C}^{*}$ and $\theta^{*}$. These multiple prediction errors $\tilde{s}_{(i)}(t)$ use parameter adaptive laws initialized with different representative initial values $\theta_{D}(0), \theta_{C}(0)$ and $\theta(0)$ to generate the multiple sets of parameter estimates $\theta_{D(i)}(t), \theta_{C(i)}(t)$ and $\theta_{(i)}(t)$ and the corresponding multiple control signals $u_{(i)}(t)$. Therefore, we use cost function $J_{(i)}(t)$ to choose a desired control signal $u(t)$ corresponding to the smallest prediction error, and a better system performance 
would be probably obtained.

To comprehensively understand the possible system performance ensured by a multiple-model adaptive controller, we consider a single-model scheme initialized with $\theta_{D}(0), \theta_{C}(0)$ and $\theta(0)$ far away from $\theta_{D}^{*}, \theta_{C}^{*}$ and $\theta^{*}$, and a multiple-model adaptive control scheme initialized with multiple $\theta_{D(i)}(0), \theta_{C(i)}(0)$ and $\theta_{(i)}(0)$, some of which can be closer to $\theta_{D}^{*}, \theta_{C}^{*}$ and $\theta^{*}$. For the single-model case, such an undesired choice of initial parameter values would lead to unsatisfying system transient and steady-state performance. For the multiple-model case, its control switching mechanism selects the control signal corresponding to the smallest prediction error, which usually results in the best performance (achieved by the best individual controller among the set of controllers), sometimes results in some sub-optimal performance (when the smallest prediction error or its cost function does not correspond to the smallest parameter uncertainty effect), or even results in the worst performance (when a smaller cost function happens to be related to a larger effect of parameter uncertainties for some time interval). However, undesired performance would also occur with a single-model adaptive controller, because the corresponding initial parameters can also have the chance to be used as the initial parameters of a single-model adaptive controller. In this sense, a multiple-model adaptive controller will not make the system transient worse, but can generally improve system performance.

\subsection{Simulation Study}

In this section, simulation results of our multiple-model adaptive control scheme are presented and discussed to verify its advantages over the single-model adaptive scheme developed in last chapter. We use the same 2-link robot manipulator model as the one used in single-model cases for comparison. As a result, the simulation system 
will not be introduced again, since the system equations, parametrization, control signal, and system parameters in this simulation study are all the same as those in last chapter. Therefore we are able to analyze different control effects between singlemodel adaptive control and multiple-model adaptive control without concern about system parameters' influences.

\subsubsection{Simulation Results}

Recall the simulation study of single-model control scheme in Chapter 3, we did simulation for two cases with different desired trajectories. In this section, we analyze the control effects of multiple-model scheme in the same two cases.

Simulation conditions. In order to test our multiple-model adaptive control design, we chose arbitrarily scattered three parameter estimators with different sets of initial values $\theta(0)$ to develop three adaptive controllers. For the first controller, the initial values are chosen as $\theta_{1}(0)=0.3, \theta_{2}(0)=0.01, \theta_{3}(0)=0.01, \theta_{4}(0)=10, \theta_{5}(0)=$ $1, \theta_{D 1}(0)=0.01, \theta_{D 2}(0)=0.01, \theta_{D 3}(0)=0.01, \theta_{C}(0)=0.01$, which are the values we used in the simulation of single-model adaptive control scheme. For the second controller, we chose the initial values as $\theta_{1}(0)=0.1, \theta_{2}(0)=1, \theta_{3}(0)=1, \theta_{4}(0)=$ $1, \theta_{5}(0)=1, \theta_{D 1}(0)=1, \theta_{D 2}(0)=1, \theta_{D 3}(0)=1, \theta_{C}(0)=1$. The initial values chosen for the third controller are $\theta_{1}(0)=0.35, \theta_{2}(0)=0.013, \theta_{3}(0)=0.012, \theta_{4}(0)=$ $11, \theta_{5}(0)=0.5, \theta_{D 1}(0)=0.35, \theta_{D 2}(0)=0.013, \theta_{D 3}(0)=0.012, \theta_{C}(0)=0.012$.

The desired trajectory was chosen as $q_{1 d}=\pi, q_{2 d}=\pi / 2$ for Case I, and $q_{1 d}(t)=$ $\pi / 3+\sin (t), q_{2 d}(t)=\pi / 6+\cos (t)$ for Case II, along with their first and second derivatives. The initial joint positions, design parameters and adaptive gains are the same as those in simulation of single-model cases: $q_{1}(0)=\pi / 6, q_{2}(0)=\pi / 3, K_{d}=I_{2}$, $\Lambda=I_{2}, \Lambda_{1}=0.8 I_{2}, \Gamma_{D}=0.1 I_{3}, \Gamma_{C}=0.1 I_{1}$, and $\Gamma=10 I_{5}$. 
For the cost function $J_{i}(t)$ defined in equation (4.16), we chose $\lambda=0.5$ for simulation. Besides, we use waiting time $T_{\min }=0.5 \mathrm{sec}$ to prevent arbitrarily fast switching.

System responses. The simulation results obtained from Case I are shown in Figures 7-10, and results obtained from Case II in Figures 11-14. From the transient performance of the multiple-model adaptive control shown in Figures 7-9 and Figures 11-13, we find that our control objective could be achieved in both cases, since the tracking errors $e(t)=q(t)-q_{d}(t)$ both converge to zero, and parameter estimation errors $\theta(t)-\theta^{*}, \theta_{D}(t)-\theta_{D}^{*}, \theta_{C}(t)-\theta_{C}^{*}$ are all bounded.

We also notice that in both cases, the control switching index $i$ switches from 1 to 3 after a short time interval, as shown in Figure 10(a) and Figure 14(a), which means the manipulator system takes advantage of the first controller at the very beginning, and then switches to the third controller because it has the smallest cost function among these controllers. Related information can be found in Figure 10(b) and Figure 14(b), from which we could find that $J_{3}$ (represented by the solid curve) always has the smallest value so that the third controller is applied to the manipulator system after the waiting time, and no more switch would happen afterwards. 


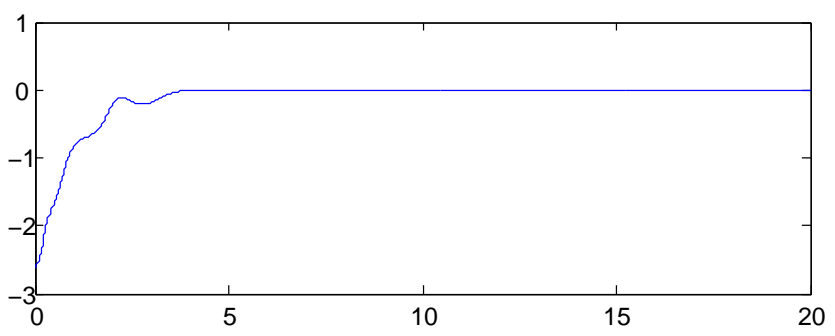

(a) Tracking error $e_{1}(t)$ in $\mathrm{rad} / \mathrm{sec}$

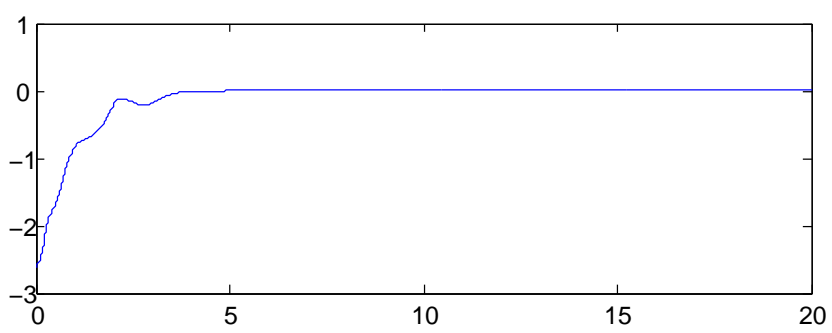

(b) Tracking error $\mathrm{e}_{2}(\mathrm{t})$ in $\mathrm{rad} / \mathrm{sec}$

Figure 4.1: Tracking errors $e(t)=q(t)-q_{d}(t)$ for the multiple-model scheme (Case I).

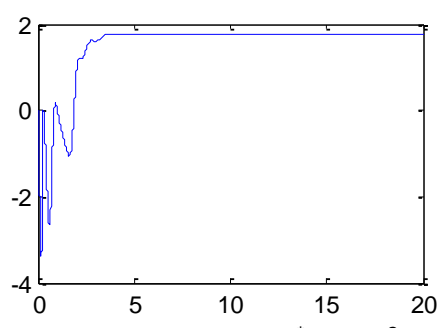

(a) Parameter error $\theta_{1(\mathrm{i})}-\theta_{1}^{*}$ in $\mathrm{kgm}^{2} / \mathrm{sec}$

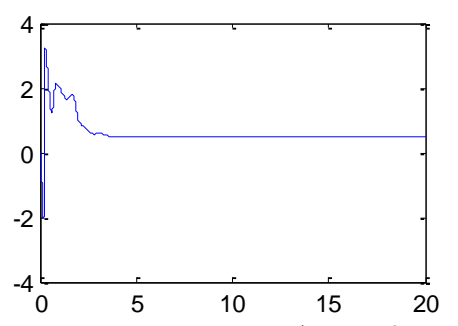

(b) Parameter error $\theta_{2(\mathrm{i})}-\theta_{2}^{*}$ in $\mathrm{kgm}^{2} / \mathrm{sec}$

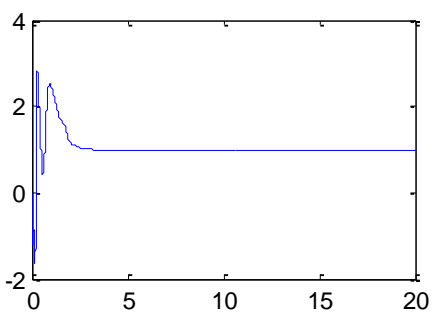

(c) Parameter error $\theta_{3(\mathrm{i})}-\theta_{3}^{*}$ in $\mathrm{kgm}^{2} / \mathrm{sec}$

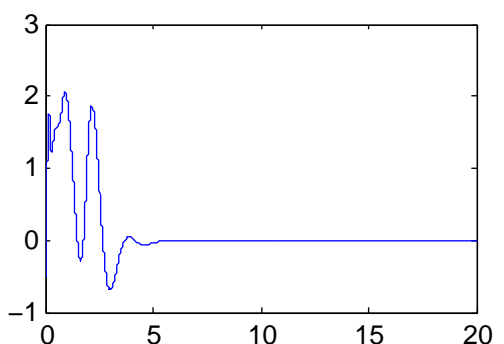

(d) Parameter error $\theta_{4(\mathrm{i})}-\theta_{4}^{*}$ in $\mathrm{kgm}^{2} / \mathrm{sec}$

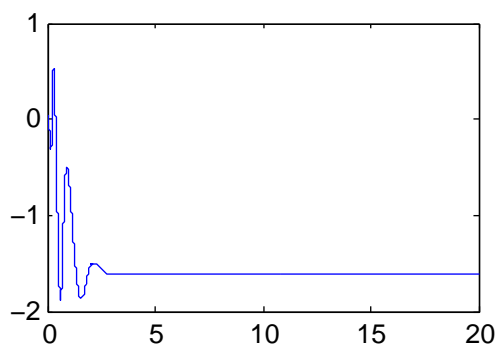

(e) Parameter error $\theta_{5(\mathrm{i})}-\theta_{5}^{*}$ in $\mathrm{kgm}^{2} / \mathrm{sec}$

Figure 4.2: Parameter errors of $\theta_{(i)}(t)$ for the multiple-model scheme (Case I). 

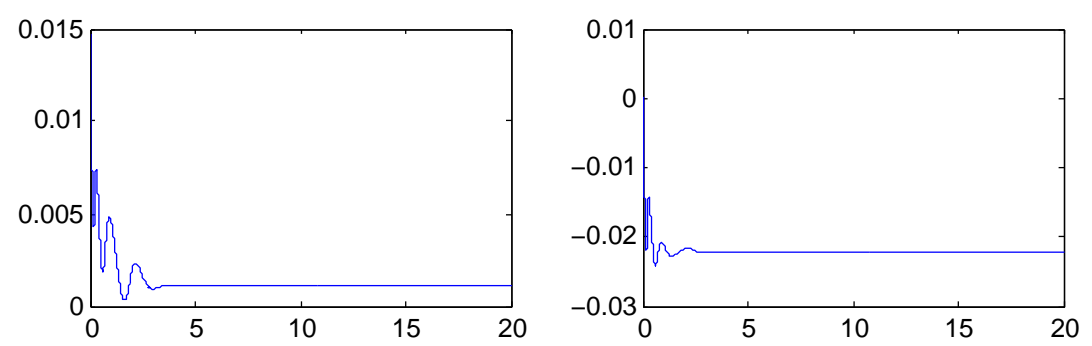

(a) Parameter error $\theta_{\mathrm{D} 1(\mathrm{i})}-\theta_{\mathrm{D} 1}^{*}$ in $\mathrm{kgm}^{2} / \mathrm{sec}$

(b) Parameter error $\theta_{\mathrm{D} 2(\mathrm{i})}-\theta_{\mathrm{D} 2}^{*}$ in $\mathrm{kgm}^{2} / \mathrm{sec}$
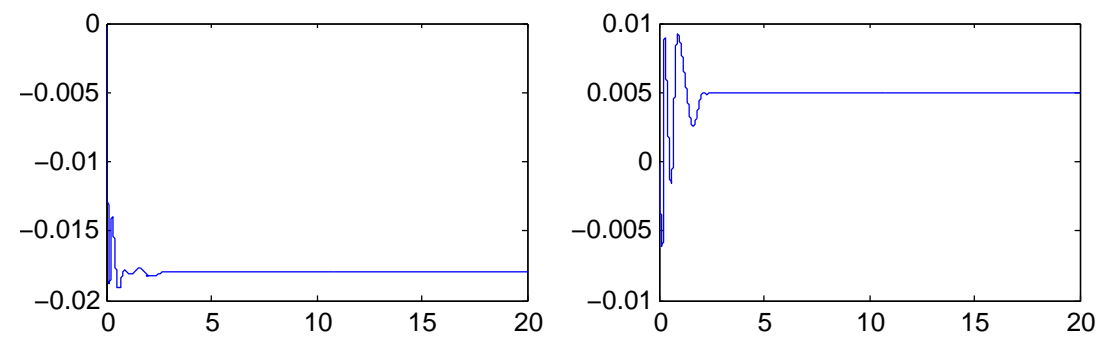

(c) Parameter error $\theta_{\mathrm{D} 3(\mathrm{i})}-\theta_{\mathrm{D} 3}^{*}$ in $\mathrm{kgm}^{2} / \mathrm{sec}$

(d) Parameter error $\theta_{\mathrm{C}(\mathrm{i})}-\theta_{\mathrm{C}}^{*}$ in $\mathrm{kgm}^{2} / \mathrm{sec}$

Figure 4.3: Parameter errors of $\theta_{D(i)}(t)$ and $\theta_{C(i)}(t)$ for the multiple-model scheme (Case I).

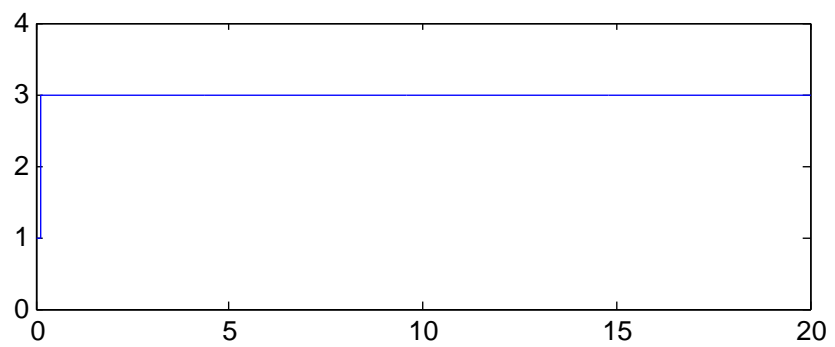

(a) Control switching index $\mathrm{i}$

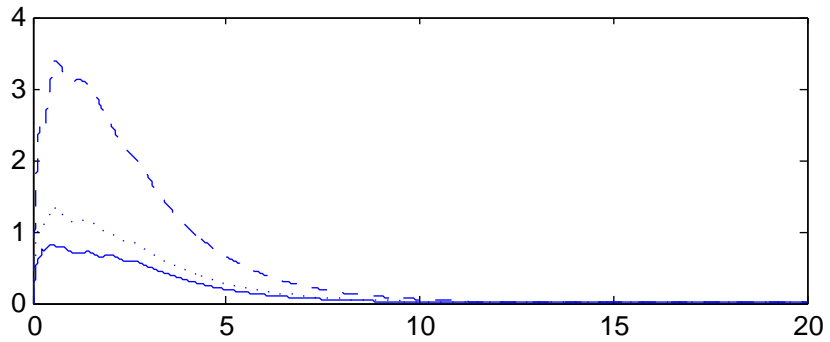

(b) Cost function $\mathrm{J}_{1}(\mathrm{t})$ (dotted), $\mathrm{J}_{2}(\mathrm{t})$ (dashed) and $\mathrm{J}_{3}(\mathrm{t})$ (solid)

Figure 4.4: Control switching index $i$ and cost functions $J_{i}(t)$ of the multiple-model scheme (Case I). 


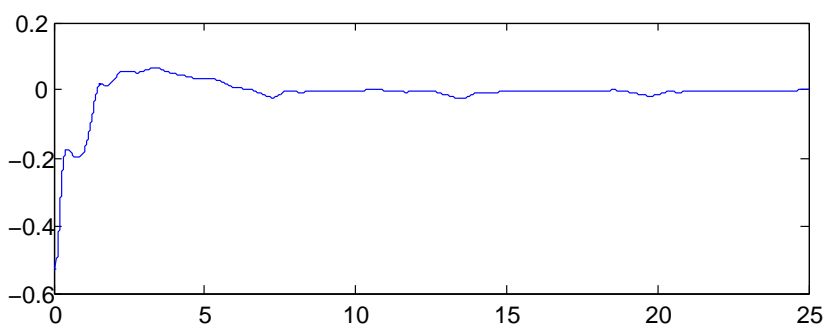

(a) Tracking error $\mathrm{e}_{\mathrm{q}}(\mathrm{t})$ in $\mathrm{rad} / \mathrm{sec}$

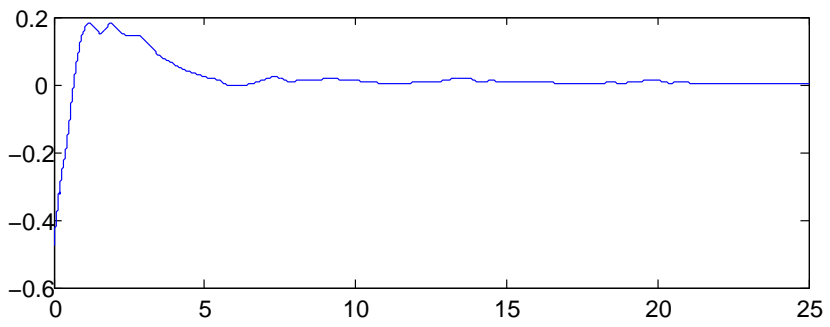

(b) Tracking error $e_{2}(t)$ in rad/sec

Figure 4.5: Tracking errors $e(t)=q(t)-q_{d}(t)$ for the multiple-model scheme (Case II).

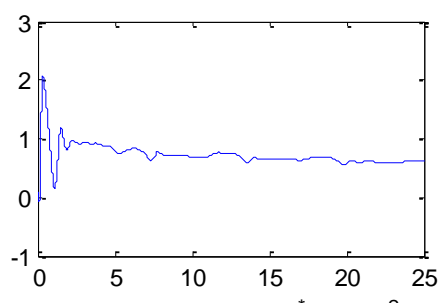

(a) Parameter error $\theta_{1(\mathrm{i})}-\theta_{1}^{*}$ in $\mathrm{kgm}^{2} / \mathrm{sec}$

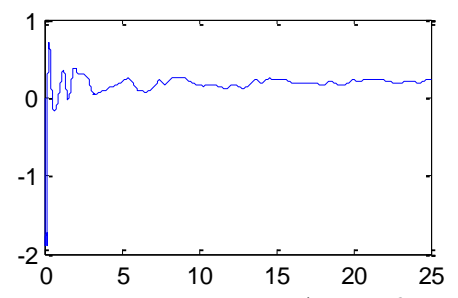

(b) Parameter error $\theta_{2(i)}-\theta_{2}^{*}$ in $\mathrm{kgm}^{2} / \mathrm{sec}$

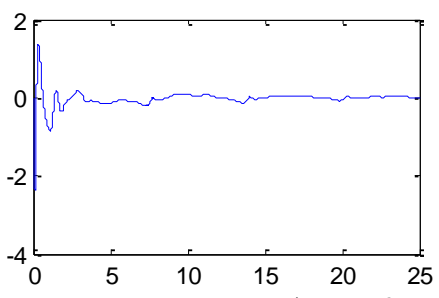

(c) Parameter error $\theta_{3(\mathrm{i})}-\theta_{3}^{*}$ in $\mathrm{kgm}^{2} / \mathrm{sec}$

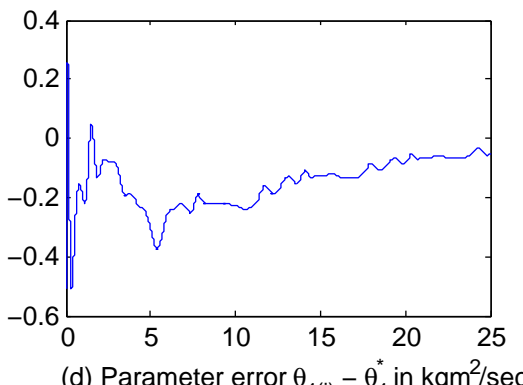

(d) Parameter error $\theta_{4(\mathrm{i})}-\theta_{4}^{*}$ in $\mathrm{kgm}^{2} / \mathrm{sec}$

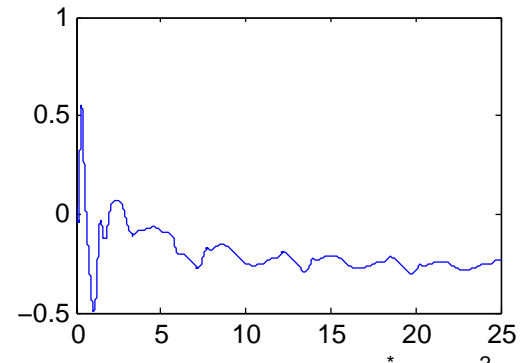

(e) Parameter error $\theta_{5(\mathrm{i})}-\theta_{5}^{*}$ in $\mathrm{kgm}^{2} / \mathrm{sec}$

Figure 4.6: Parameter errors of $\theta_{(i)}(t)$ for the multiple-model scheme (Case II). 

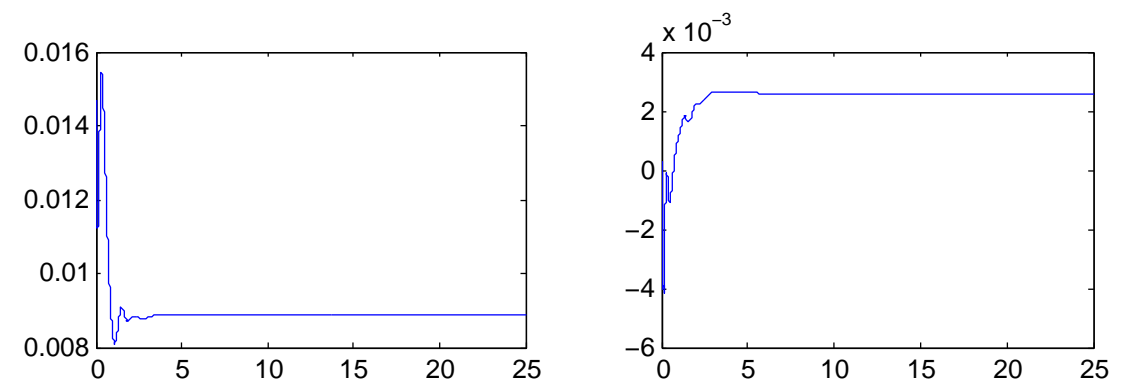

(a) Parameter error $\theta_{\mathrm{D} 1(\mathrm{i})}-\theta_{\mathrm{D} 1}^{*}$ in $\mathrm{kgm}^{2} / \mathrm{sec}$

(b) Parameter error $\theta_{\mathrm{D} 2(\mathrm{i})}-\theta_{\mathrm{D} 2}^{*}$ in $\mathrm{kgm}^{2} / \mathrm{sec}$
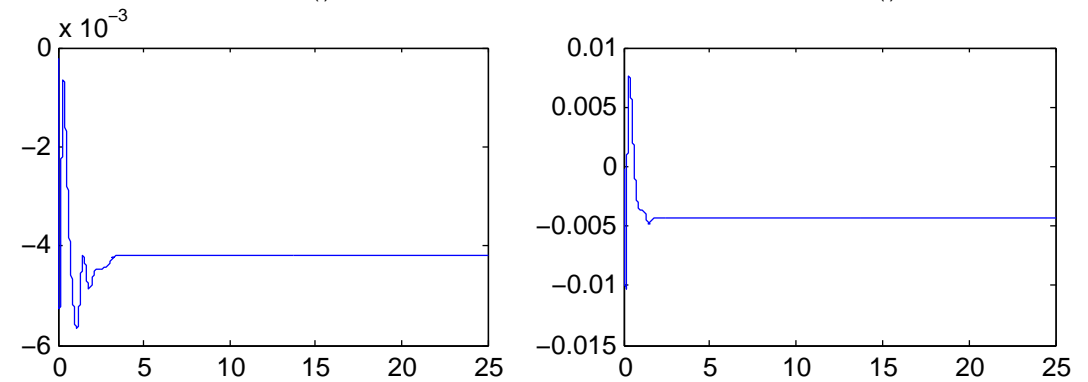

(c) Parameter error $\theta_{\mathrm{D} 3(\mathrm{i})}-\theta_{\mathrm{D} 3}^{*}$ in $\mathrm{kgm}^{2} / \mathrm{sec}$

(d) Parameter error $\theta_{\mathrm{C}(\mathrm{i})}-\theta_{\mathrm{C}}^{*}$ in $\mathrm{kgm}^{2} / \mathrm{sec}$

Figure 4.7: Parameter errors of $\theta_{D(i)}(t)$ and $\theta_{C(i)}(t)$ for the multiple-model scheme (Case II).

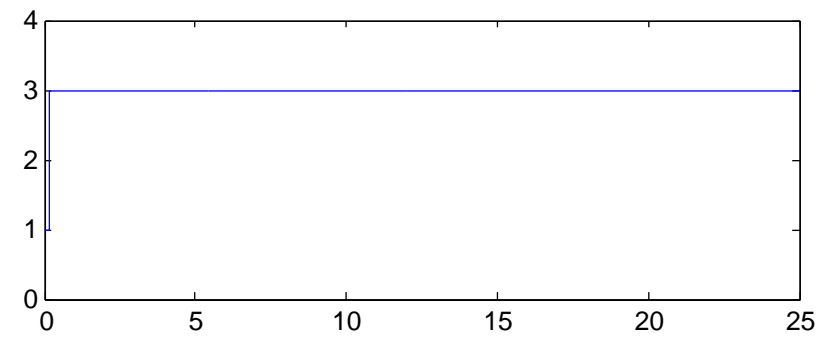

(a) Control switching index $\mathrm{i}$

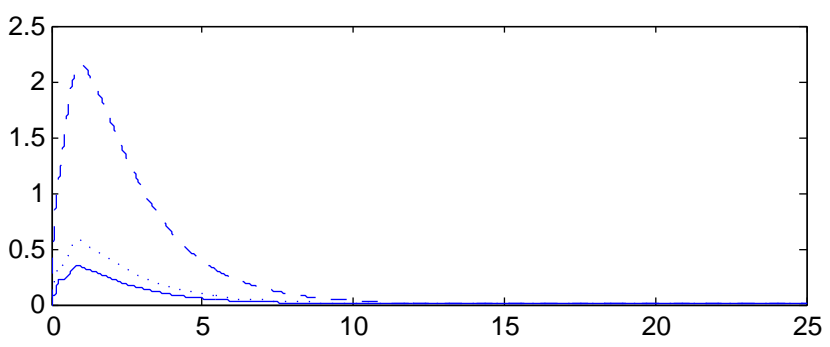

(b) Cost function $\mathrm{J}_{1}(\mathrm{t})$ (dotted), $\mathrm{J}_{2}(\mathrm{t})$ (dashed) and $\mathrm{J}_{3}(\mathrm{t})$ (solid)

Figure 4.8: Control switching index $i$ and cost function $J_{i}(t)$ of the multiple-model scheme (Case II).

Discussion. Since the initial joint positions and adaptive gains in multiple-model 
control scheme are chosen to be the same as those in single-model control scheme for every controller, a reliable comparison could be made. For Case I, it takes around 10 seconds for tracking errors with single-model scheme to converge to zero, while with multiple-model scheme, it only takes around 4 seconds. Overshoot in tracking errors is avoided as well with multiple-model scheme. For Case II, trajectory tracking and parameter convergence are achieved more quickly, and a much smaller overshoot could also be observed with multiple-model scheme. To conclude, significant performance improvements are obtained in both cases compared with the single-model scheme.

These simulation results agree with our assumption in Chapter 4 that smaller prediction errors probably mean smaller parameter errors, and smaller parameter uncertainties and errors lead to better system performance.

\subsubsection{Additional Simulation Results}

Since the cost function is based on the prediction errors, the control switching mechanism would select the control signal corresponding to the smallest prediction errors. However, as we mentioned before, the selected control signal is probably, but not certainly the one which could lead to the best performance. In the simulation study of multiple-model scheme, we did meet undesirable results in some cases. One of them is presented as follows to illustrate this uncertainty of multiple-model scheme.

Simulation conditions. The three sets of initial values for controllers in this case are chosen as following: $\theta_{1}(0)=0.3, \theta_{2}(0)=0.01, \theta_{3}(0)=0.01, \theta_{4}(0)=10$, $\theta_{5}(0)=1, \theta_{D 1}(0)=0.01, \theta_{D 2}(0)=0.01, \theta_{D 3}(0)=0.01, \theta_{C}(0)=0.01$ for the first one; $\theta_{1}(0)=1, \theta_{2}(0)=1, \theta_{3}(0)=1, \theta_{4}(0)=1, \theta_{5}(0)=1, \theta_{D 1}(0)=1, \theta_{D 2}(0)=1, \theta_{D 3}(0)=$ $1, \theta_{C}(0)=1$ for the second one; $\theta_{1}(0)=0.35, \theta_{2}(0)=0.013, \theta_{3}(0)=0.012, \theta_{4}(0)=$ $11, \theta_{5}(0)=0.5, \theta_{D 1}(0)=0.35, \theta_{D 2}(0)=0.013, \theta_{D 3}(0)=0.012, \theta_{C}(0)=0.012$ for the 
third one. Choose the desired trajectory in Case II: $q_{1 d}(t)=\pi / 3+\sin (t), q_{2 d}(t)=$ $\pi / 6+\cos (t)$. The other conditions are exactly the same as those in Case I and II.

System responses. The tracking errors $e(t)$, control switching index $i$ and cost functions $J_{i}(t)$ are shown in Figures 15-16. We find that the system use the second controller after a short time interval. This is because the cost function $J_{2}$ becomes the smallest one in this case, as shown in Figure 16(b). However, the transient performance of multiple-model scheme in this case is not better than single-model scheme, which means the smallest prediction errors may not lead to the best transient performance, but at least, multiple-model control will not perform worse than the worst controller.
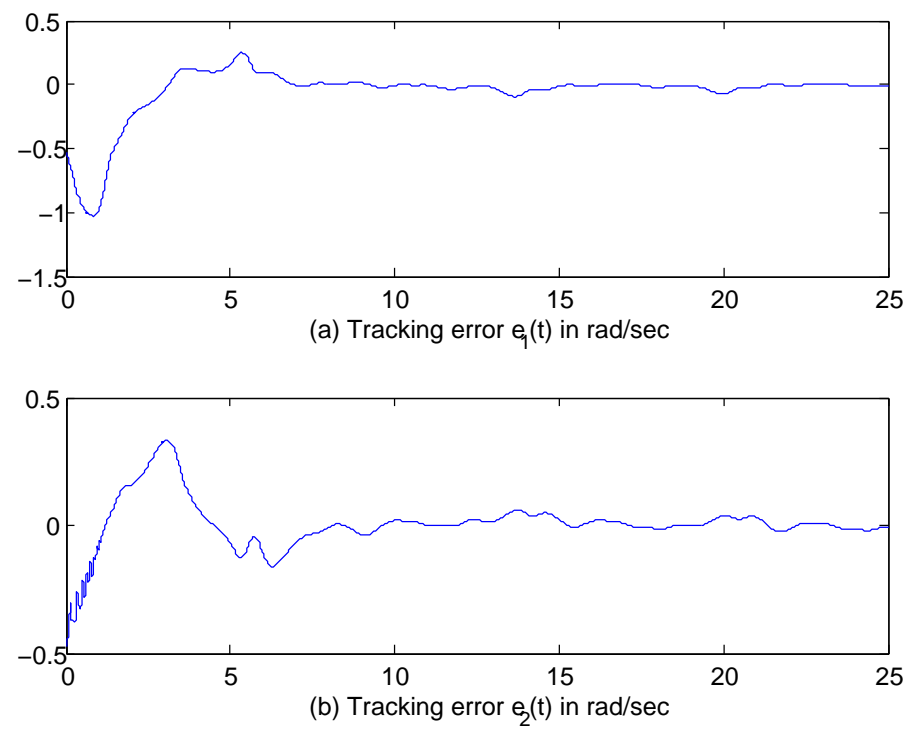

Figure 4.9: Tracking error $e(t)=q(t)-q_{d}(t)$ for multiple-model scheme case III. 


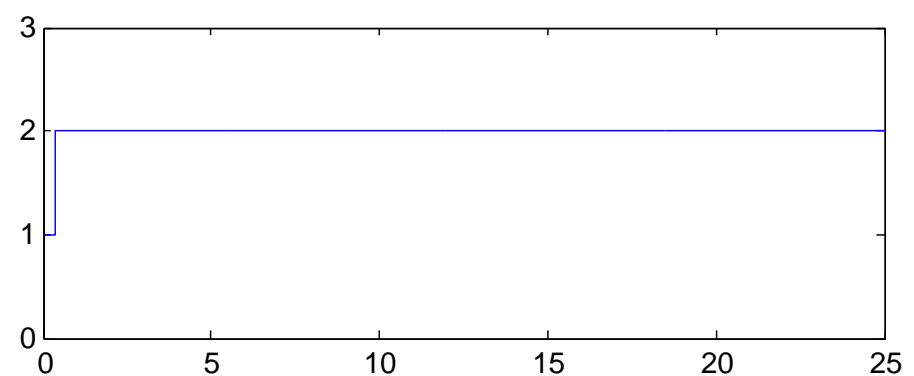

(a) Control switching index $\mathrm{i}$

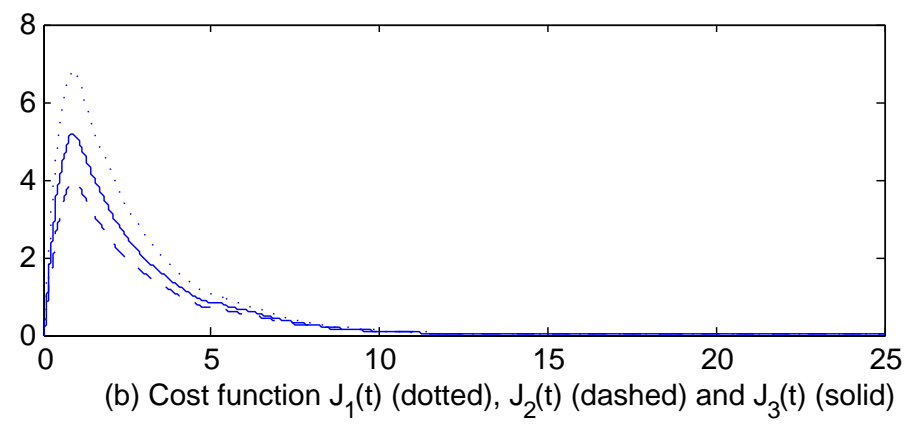

Figure 4.10: Control switching index $i$ and cost function $J_{i}(t)$ of multiple-model scheme case III. 


\section{Chapter 5}

\section{Conclusions and Future Work}

\subsection{Conclusions}

The multiple-model adaptive control scheme proposed in this thesis is a promising method to solve the control problem of robotic manipulators, and to improve its overall tracking performance. The work presented here is a complete study of a general control methodology, which strictly consists of prediction, adaptive laws, control input signals, stability analysis. It represents an attempt to demonstrate that switching between multiple models can be used effectively for performance improvement in trajectory-tracking tasks of robotic manipulators.

The algorithms introduced in Chapter 2 briefly summarize the main contributions in the field of adaptive robot control, as well as the basic concepts of multiple-model control. The drawbacks and limits of these existing algorithms motivated us to generate a novel control scheme to solve or avoid these problems.

The controller we designed in Chapter 3 is based on the dynamic prediction error, with a parameter projection scheme proposed to verify the stability of this adaptive control algorithm. This new scheme can be used as a basic structure for multiple- 
model adaptive control design, and the development and expansion are stated in Chapter 4.

The multiple-model control scheme makes use of multiple prediction signals of the manipulator and a switching scheme that determines the specific torque input to drive the joint actuators. The main idea is to generate a torque vector that is based on a model that best approximates the manipulator dynamics at every instant. The global system stability could be obtained under the switching rule, which provides demonstration of the effectiveness of our algorithm. Therefore, we conclude that this multiple-model adaptive control scheme is possible to achieve desired performance improvement.

Simulation studies on a two-link robotic manipulator were also carried out in both Chapter 3 and Chapter 4 . The results confirmed those properties predicted by the theoretical analysis, and demonstrated that the proposed algorithm improves the overall tracking performance over the single-model-based adaptive controller.

Consequently, the problem stated in Section 2.5, which is to design a control scheme for robotic manipulators to achieve trajectory tracking with desired performance, can be solved by the combination of adaptive control and multiple-model control.

\subsection{Future Research Topics}

Adaptive multiple-model switching control of robotic manipulators is a promising area to solve undesired transient performance, as discussed in this thesis. However, this research area is not mature enough, and challenges still exist, needing to be overcome. In this section, we introduce several potential extensions as future research topics of our research. 
Guarantee of desired system performance. The multiple-model adaptive control scheme proposed in Chapter 4 is based on the assumption that a smaller prediction error leads to smaller system parameter uncertainties, which would correspond to better system performance. However, this assumption is not always true, which means the multiple-model adaptive control cannot guarantee performance improvement in every situation. Therefore, it is worth investigating whether there would be a better way to quantitatively measure the parameter uncertainties so that optimal control effect will always be achieved using multiple-model control. There are several researchers preferred composite adaptive control for robot manipulators, where both tracking error and prediction error are used to reflect the parameter uncertainty [34], [35]. This may be a feasible method for enhancement of our control scheme.

Multi-layer multiple-model control design. The control scheme proposed in this thesis can be further expanded to a multi-layer multiple-model control design, which uses multiple groups of adaptive controllers for further improving system performance. Each group is designed from expanding one basic controller to a set of controllers initialized from different parameter subregions (which we have already done in this thesis), or to a different set of controllers update by different adaptation gains (which has been discussed but not simulated in this thesis), or to a combination of both groups of controllers to form a two-layer multiple-model control scheme (which has not been constructed in this thesis). By a control switching mechanism selecting the control input signal corresponding to the minimal performance index from much more multiple control signals, faster and more steady transient response would probably be achieved.

This thesis intends to lay the groundwork for multiple-model adaptive control 
scheme for robotic manipulators. It would be significant if more research will be done in this potential area, strengthening the framework of adaptive control methodology. 


\section{Bibliography}

[1] Aly, G., A. Badr, and Z. Binder, "Multi-model control of MIMO systems: location and control algorithms," International journal of systems science vol. 19, no. 9, pp. 1687-1698, 1988.

[2] Badr A., Z. Binder, R. PERRET, et al, "A multi-model tracking controller for distributed control system," A bridge between control science and technology, vol. 5, pp. 2823-2828, 1985.

[3] Badr, A., Z. Binder, and D. Rey, "Application of tracking multimodel control to a non-linear thermal process," International journal of systems science, vol. 21, no. 9, pp. 1795-1803, 1990.

[4] Berghuis, H., R. Ortega, and H. Nijmeijer, "A robust adaptive robot controller," IEEE Transactions on Robotics and Automation vol. 9, no.3, pp. 825-830, 1993.

[5] Ciliz, M. K. and K. S. Narendra, "Multiple model based adaptive control of robotic manipulators," Proceedings of the 33th IEEE Conference on Decision and Control, vol. 2, pp. 1305-1310, 1994.

[6] Ciliz, M. K. and K. S. Narendra, "Intelligent control of robotic manipulators: a multiple model based approach," iros. IEEE, pp. 2422, 1995. 
[7] Ciliz, M. K. and K. S. Narendra, "Adaptive control of robotic manipulators using multiple models and switching," The International journal of robotics research, vol. 15, no. 6, pp. 592-610, 1996.

[8] Ciliz, M. K. "Combined direct and indirect adaptive control of robot manipulators using multiple models," Advanced Robotics, vol. 20, no. 4, pp. 483-497, 2006.

[9] Craig, J. J., P. Hsu, and S.S. Sastry, "Adaptive control of mechanical manipulators," IEEE International Conference on Robotics and Automation, vol.3, pp.190$195,1986$.

[10] Craig, J. J., P. Hsu, and S. S. Sastry, "Adaptive control of mechanical manipulators," The International Journal of Robotics Research, vol.6, no.2, pp. 16-28, 1987.

[11] Craig, J. J., Introduction to Robotics, New York, Addison-Wesley, 1986.

[12] Fu, M., and B. R. Barmish, "Adaptive stabilization of linear systems via switching control," IEEE Transactions on Automatic Control, vol. 31, no. 12, pp. 1097$1103,1986$.

[13] Ghorbel, F., B. Srinivasan, and M. W. Spong, "On the uniform boundedness of the inertia matrix of serial robot manipulators," Journal of Robotic Systems, vol. 15, no. 1, pp. 17-28, 1998.

[14] Kelly, R., R. Carelli, M. Amestegui and R. Ortega, "An adaptive impedance control of robot manipulator," IEEE Conference on Robotics and Automation, pp. 572-577, 1987. 
[15] Kelly, R., and R. Carelli, "Unified approach to adaptive control of robotic manipulators," IEEE Conference on Decision and Control, pp. 1598-1603, 1988.

[16] Koditschek, D. E., “Adaptive techniques for mechanical systems," Yale Workshop on Applications of Adaptive Systems Theory, pp. 259-265, 1987.

[17] Lee, C., "Adaptive control of a class of nonlinear systems using multiple parameter models," International Journal of Control Automation and Systems, vol. 4, no. 4, pp. 428, 2006.

[18] Levin, A. U., and K. S. Narendra, "Control of nonlinear dynamical systems using neural networks: Controllability and stabilization," IEEE Transactions on Neural Networks, vol. 4, no. 2, pp. 192-206, 1993.

[19] Levin, A. U., and K. S. Narendra. "Control of nonlinear dynamical systems using neural networks. II. Observability, identification, and control," IEEE Transactions on Neural Networks, vol. 7, no. 1, pp. 30-42, 1995.

[20] Lewis, F. L., C. T. Abdallah, and D. M. Dawson, Control of Robot Manipulators, New York, Macmillan, 1993.

[21] Li, W., J. J. E. Slotine, "An indirect adaptive robot controller," Systems and control letters, vol. 12, no. 3, pp. 259-266, 1989.

[22] Middleton, R. H., G. C. Goodwin, D. J. Hill, and D. Q. Mayne, "Design issues in adaptive control," IEEE Transactions on Automatic Control, vol. 33, no. 1, pp. 50-58, 1988.

[23] Middleton, R. H. and G. C. Goodwin, "Adaptive computed torque control for rigid link manipulations," Systems and Control Letters, vol. 10, no. 1, pp. 9-16, 1988. 
[24] Narendra, K. S., J. Balakrishnan, and K. M.Ciliz, "Adaptation and learning using multiple models, switching, and tuning," IEEE Control Systems, vol. 15, no. 3, pp. 37-51, 1995.

[25] Narendra, K, S., and J. Balakrishnan, "Improving transient response of adaptive control systems using multiple models and switching," IEEE Transactions on Automatic Control, vol. 39, no. 9 pp. 1861-1866, 1994.

[26] Narendra, K. S., and J. Balakrishnan, "Intelligent control using fixed and adaptive models," Proceedings of IEEE 33rd Conference Decision and Control, 1 vol. 2, pp. 1680-1685, 1994.

[27] Narendra, K. S., and K. Parthasarathy, "Identification and control of dynamical systems using neural networks," IEEE Transactions on Neural Networks, vol. 1, no. 1, pp. 4-27, 1990.

[28] Narendra, K. S., and C. Xiang, "Adaptive control of discrete-time systems using multiple models," Proceedings of the 37th IEEE Conference on Decision and Control, vol. 4, pp. 3978-3983, 1998.

[29] Narendra, K. S., and C. Xiang, "Adaptive control of discrete-time systems using multiple models," IEEE Transactions on Automatic Control, vol. 45, no. 9, pp. 1669-1686, 2000.

[30] Oliver, J. P. O., O. A. Domnguez-Ramirez, and E. S. E. Quezada, "Trajectory tracking control for robotics manipulators based on passivity," Electronics, Robotics and Automotive Mechanics Conference, pp. 484-489, IEEE, 2008.

[31] Ortega, R., and M.W. Spong, "Adaptive motion control of rigid robots: a tutorial," Automatica, vol. 25, no. 6, pp. 877-888, 1988. 
[32] Rugthum, T., and G. Tao, "An adaptive actuator failure compensation scheme for a cooperative manipulator system," Robotica, vol. 34, no. 7, pp. 1529-1552, 2016.

[33] Slotine, J. J. E., and W. Li, "Adaptive Manipulator Control", The American Society of Mechanical Engineers Winter Annual Meeting, Anaheim, CA. 1986.

[34] Slotine, J. J. E. and W. Li, "On the adaptive control of robot manipulators," International Journal of Robotics Research, vol. 6, no. 3, pp. 49-59, 1987.

[35] Slotine, J. J. E. and W. Li, "Adaptive strategies in constrained manipulation," IEEE International Conference on Robotics and Automation, pp. 595601, 1987.

[36] Slotine, J. J. E., and W. Li, "Adaptive manipulator control: A case study," IEEE Transactions on Automatic Control, vol. 33, no.11, pp. 995-1003, 1988.

[37] Spong M. W., and M. Vidyasagar, Robot Dynamics and Control, John Wiley \& Sons, New York, 1989.

[38] Tao, G., Adaptive Control Design and Analysis, John Wiley \& Sons, Hoboken, NJ, 2003.

[39] Tan, C., X. Yao, G. Tao, and R. Qi, "A Multiple-Model Based Adaptive Actuator Failure Compensation Scheme for Control of Near-Space Vehicles," IFAC Proceedings Volumes, vol. 45, no. 20, pp. 594-599, 2012.

[40] Tan, C., G. Tao, and R. Qi, "A discrete-time parameter estimation based adaptive actuator failure compensation control scheme," International Journal of Control, vol. 86, no.2, pp.276-289, 2013. 
[41] Tomei, P., "Robust adaptive friction compensation for tracking control of robot manipulators," IEEE Transactions on Automatic and Control, vol. 45, no. 11, pp. 2164-2169, 2000.

[42] Whitcomb, L. L., A. Rizzi, and D. E. Koditschek, "Comparative Experiments with a New Adaptive Controller for Robot Arms," IEEE Transactions on Robotics and Automation, vol. 9, no.1, pp. 59-70, 1993. 